\title{
BODY-RELATED EMOTIONAL EXPERIENCES OF YOUNG ABORIGINAL WOMEN
}

\author{
A Thesis Submitted to the College of \\ Graduate Studies and Research \\ in Partial Fulfillment of the Requirements \\ for the Degree of Master of Science \\ in the College of Kinesiology \\ University of Saskatchewan \\ Saskatoon
}

By

Tara-Leigh Fleming

(C) Copyright Tara-Leigh Fleming, September 2004. All rights reserved. 


\title{
PERMISSION TO USE
}

In presenting this thesis in partial fulfillment of the requirements for a Postgraduate degree from the University of Saskatchewan, I agree that the Libraries of this University may make it freely available for inspection. I further agree that permission for copying of this thesis in any manner, in whole or in part, for scholarly purposes may be granted by the professor or professors who supervised my thesis work or, in their absence, by the Head of the Department or the Dean of the College in which my thesis work was done. It is understood that any copying or publication or use of this thesis or parts thereof for financial gain shall not be allowed without my written permission. It is also understood that due recognition shall be given to me and to the University of Saskatchewan in any scholarly use which may be made of any material in my thesis.

Requests for permission to copy or to make other use of material in this thesis in whole or part should be addressed to:

\author{
Dean of the College of Kinesiology \\ University of Saskatchewan \\ Saskatoon, Saskatchewan
}

S7N 5B3 


\begin{abstract}
The purpose of this qualitative case study was to provide insight into the bodyrelated emotional experiences of young Aboriginal women. Four young women took part in this study; three who identified themselves as Aboriginal (one 14-year-old, two 18-year-olds) and one who identified herself as non-Aboriginal (18-year-old). An important strength of this study was that the young women were part of an intact group. The young women were members of a mentorship group at their local high school and this mentorship group was for young women who had faced adversity in their lives.

The intent of this study was to listen to the stories and experiences of young women in order to better understand the complex nature of their body-related emotion. Feminist perspective was used to guide the study because it is a voice-centered approach based on listening to women's experiences. Cognitive-Motivational-Relational Theory was used to ground the study because it recognizes that emotion is a complex and context driven process.
\end{abstract}

Through the use of multiple methods (i.e., focus group, one-on-one interviews, artwork) the young women were able to provide an in-depth view of their experiences. Stake's (1995) guidelines for case study data analysis were followed, and the collective story of the young women's body-related emotional experiences have been described. This study contributes to the literature on the body-related emotional experiences of young Aboriginal women in a number of ways. The young women in this study possess 
many of the positive attributes (i.e., confidence, optimism) that have been associated with resiliency. Also, the emotions that were experienced by the young women were very complex and dependent upon specific contexts. The five themes that emerged from the data were conflicting cultures, need to belong, personal identity, journey to acceptance, and the body affects everything. Overall, the young women in this study noted a general level of body satisfaction, which is inconsistent with previous research surrounding young women's body-related emotion. One of the most important findings from this study is that the body-related emotional experiences of young Aboriginal women are not as negative as previous research has lead us to believe. 


\section{ACKNOWLEDGEMENTS}

I would like to thank my advisor Dr. Kent Kowalski for his enduring support and guidance throughout this research process. I would also like to thank my committee members, Dr. Adam Baxter-Jones, Martin Cannon, and Dr. Louise Humbert, and my external advisor, Dr. Linda Wason-Ellam, for their many contributions throughout this

journey. In addition, I would like to recognize the Community and Population and Health Research Training Program and the Social Sciences and Humanities Research Council of Canada for the funding I received.

I would also like to thank the four young women who participated in this study; the sharing of their experiences and stories has been invaluable. 


\section{DEDICATION}

I would like to dedicate this thesis to my parents, Peter and Cathy Fleming, for their continued love and support and for being my life long educators. 


\section{TABLE OF CONTENTS}

PERMISSION TO USE $\quad$ i

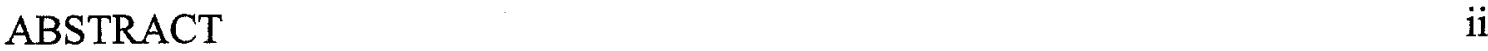

ACKNOWLEDGEMENTS . iv

DEDICATION $\quad \mathrm{v}$

TABLE OF CONTENTS

LIST OF APPENDICES IX

DEFINITIONS $\quad x$

CHAPTER 1

1.1 INTRODUCTION

1.2 REVIEW OF LITERATURE

1.2.1 The Health of Young Aboriginal Women 3

1.2.2 Body-related Emotional Experiences of Young Aboriginal Women 6

1.2.3 Positive Body-related Experiences 9

$\begin{array}{ll}\text { 1.2.4 Consistency Among All Young Women } & 11\end{array}$

1.2.5 Limitations to Previous Research with Young Aboriginal Women 16

$\begin{array}{ll}\text { 1.2.6 Feminist Perspective } & 20\end{array}$

1.2.7 Conceptual Framework $\quad 26$

1.3 STATEMENT OF THE PURPOSE 29

$\begin{array}{ll}\text { CHAPTER } 2 & 31\end{array}$ 
2.1 METHODOLOGY 31

$\begin{array}{ll}\text { 2.1.1 Case Study Approach } & 31\end{array}$

2.1.2 Data Collection 33

2.1.2.1 Personal Story $\quad 35$

2.1.2.2 Special Considerations 38

2.1.2.3 Phase One: Participant Selection 39

2.1.2.3.1 Group Profile $\quad 42$

2.1.2.4 Phase Two: Rapport Building $\quad 44$

2.1.2.5 Phase Three: Focus Group $\quad 47$

2.1.2.6 Phase Four: One-on-one Interviews

2.1.2.7 Phase Five: Art Project $\quad 53$

2.1.3 Data Analysis $\quad 54$

$\begin{array}{ll}\text { 2.1.4 Verification } & 57\end{array}$

2.1.4.1 Prolonged Time $\quad 58$

$\begin{array}{lr}\text { 2.1.4.2 Triangulation } & 59\end{array}$

$\begin{array}{ll}\text { 2.1.4.3 Member-checking } & 60\end{array}$

$\begin{array}{ll}\text { 2.1.4.4 Rich, Thick Description } & 60\end{array}$

$\begin{array}{ll}\text { 2.1.4.5 Critique Checklist } & 61\end{array}$

$\begin{array}{lr}\text { CHAPTER } 3 & 62\end{array}$

3.1 RESULTS

$\begin{array}{ll}\text { 3.1.1 Conflicting Cultures } & 62\end{array}$

$\begin{array}{ll}\text { 3.1.2 Need To Belong } & 67\end{array}$

$\begin{array}{lr}\text { 3.1.3 Personal Identity } & 69\end{array}$

3.1.4 Journey to Acceptance $\quad 71$

vii 
3.1.5 The Body Affects Everything 74

$\begin{array}{lr}3.2 \text { DISCUSSION } & 78\end{array}$

$\begin{array}{ll}\text { 3.2.1 Main Findings } & 79\end{array}$

$\begin{array}{ll}\text { CHAPTER } 4 & 92\end{array}$

4.1 SUMMARY AND CONCLUSIONS 92

4.1.1 Limitations 93

4.1.2 Recommendations for Future Research 98

$\begin{array}{ll}\text { REFERENCES } & 102\end{array}$

$\begin{array}{ll}\text { APPENDICES } & 114\end{array}$ 


\section{LIST OF APPENDICES}

$\begin{array}{ll}\text { APPENDIX A Ethical Approval } & 115\end{array}$

$\begin{array}{ll}\text { APPENDIX B Consent Form } & 117\end{array}$

APPENDIX C Interview Guide: Reaction to Media Images 121

APPENDIX D Interview Guide: Focus Group 123

APPENDIX E Interview Guide: One-on-one Interviews 125

$\begin{array}{ll}\text { APPENDIX F Participant Artwork } & 128\end{array}$

$\begin{array}{ll}\text { APPENDIX G Interview Guide: Artwork } & 131\end{array}$

APPENDIX H Critique Checklist for a Case Study Report 133 


\section{DEFINITIONS}

Aboriginal: a term that encompasses those individuals who identify themselves as legal or status Indian, non-status Indian, Inuit, and Métis (Frideres \& Gadacz, 2001).

Body mass index (BMI): is a commonly used measure of overweight and obesity and it is defined as weight in kilograms divided by height in meters squared $\left(\mathrm{kg} / \mathrm{m}^{2}\right.$; Centers for Disease Control, 2000).

Case Study: the study of the complexity of a single case and coming to understand the activity of the case within important circumstances (Stake, 1995).

Culture: "the customary beliefs, social forms, and material traits of a racial, religious, or social group" (Sarto, 1998, p.7).

Ethnicity: "a group that is socially distinguished or set apart, by others and/or by itself, primarily on the basis of cultural or national characteristics" (Ashcroft, Griffiths, \& Tiffin, 2000, p.81).

Overweight: body mass index (BMI) is at or above the $85^{\text {th }}$ percentile on genderspecific BMI-for-age growth charts developed by the Centers for Disease Control (2000).

Race: "a class or kind of people unified by a community of interests, habits, or characteristics" (Sarto, 1998, p.7). 


\section{CHAPTER 1}

\subsection{INTRODUCTION}

Relatively little literature has examined the body-related emotional experiences of young Aboriginal women. Although researchers have recently been interested in the body-related experiences of young women in general, most of the literature has typically involved young non-Aboriginal women. However, young Aboriginal women may be of increased concern because they tend to be more overweight than young white women and Aboriginal youth experience more mental illness than youth of other ethnicities (MacMillan, MacMillan, Offord, \& Dingle, 1996; Story, French, Resnick, \& Blum, 1995). When examining weight and its associated concerns together, researchers have shown that those individuals who are overweight tend to experience more concerns relating to the body (Rinderknecht \& Smith, 2002).

The few studies that have examined the body-related emotional experiences have found that many young Aboriginal women are dissatisfied with their body (Gittelshohn et al., 1996; Neumark-Sztainer, Story, Resnick, \& Blum, 1997; Story et al., 1994). Furthermore, concerns surrounding the body have been associated with numerous health compromising behaviours (e.g., chronic dieting; Neumark-Sztainer, Story, French, et al., 1997). The majority of the research that has examined the body-related emotional experiences of young Aboriginal women has focused on the negative emotions. However, there have been a few studies that have examined the more positive 
diabetes and cardiovascular disease; Flegal, Carroll, Ogden, \& Johnson, 2002). These health implications have also been recognized by the Mi'kmaq Health Research Group (1999) who indicated that young Aboriginal women feel that being overweight is one of the most important health problems affecting them.

Although physical health has been the focus of many studies, young Aboriginal women appear to be experiencing mental health issues. MacMillan et al. (1996) found that American Indian youth experience more mental health problems than their peers of other ethnic backgrounds. Similarly, McIntyre et al. (2001) have indicated that after grade two Aboriginal youth experience a continuous decrease in self-esteem, selfconcept, and self-confidence. However, there appears to be an opposite trend (i.e., increase in self-esteem) when examining youth within mainstream white society. Nevertheless, Kirmayer, Brass, and Tait (2000) have indicated that low levels of selfesteem are likely closely related to depression and anxiety, and therefore, plays an important role in overall health.

The above-mentioned studies, which have identified high levels of mental illness among Aboriginal peoples, are consistent with the RCAP (1996) who have stated that many Aboriginal people suffer from emotional ill health. We know that Aboriginal youth are experiencing mental health issues, and young Aboriginal women may be experiencing even more mental health issues when their weight is considered. Rosen et al. (1988) found that Aboriginal women who are overweight are more likely to have concerns regarding their weight compared to those who are not overweight. Furthermore, Neumark-Sztainer, Story, Resnick, et al. (1997) found that moderately overweight young Aboriginal women have been shown to have poorer emotional wellbeing than non-overweight young Aboriginal women. 
Although the emotional well-being of young Aboriginal women is comprised of many components, the physical-self has been linked to emotional well-being in numerous ways. First, Cummins, Ireland, Resnick, and Blum (1999) have stated that one of the most important predictors of self-esteem is satisfaction with the physical-self. Second, the pride individuals have about their body (i.e., body pride) has also been positively associated with overall emotional health, and is the strongest predictor of emotional health, for female Aboriginal adolescents (Cummins et al., 1999). Third, moderately overweight Aboriginal girls may experience higher rates of suicide ideation than those girls who are not overweight or who are very overweight, thus indicating there is a link between mental health and the physical-self (Neumark-Sztainer, Story, Resnick, et al., 1997). Therefore, it is possible that the emotional health of young Aboriginal women could be at stake because overweight has been associated with poor emotional well-being and the literature states that many young Aboriginal women are overweight.

In addition to the above-mentioned health implications that are associated with the physical-self, concerns with body appearance, and the negative emotions that accompany these concerns, have been associated with depression, decreased self-worth, smoking, dietary restraint, and avoidance of physical activity (French, Perry, Leon, \& Fulkerson, 1994; Friedstad \& Klepp, 1997; Harter, 1999; Leary, 1995; Page, Ashford, Biddle, \& Fox, 1993; Page \& Fox, 1997). Therefore, if a young woman is not satisfied with her body, she may experience other potentially detrimental health effects.

As with research from many disciplines, the majority of the research surrounding body issues has involved primarily Caucasian women. However, recent evidence suggests that young Aboriginal women are experiencing many issues related to their 
bodies (Neumark-Sztainer, Story, French, et al., 1997; Neumark-Sztainer, Story, Resnick, et al., 1997; Rosen et al., 1988; Story et al., 1995; Story et al., 1994). Understanding issues surrounding the body is important, and, as the RCAP (1996) has indicated, for many Aboriginal cultures "good health is a state of balance and harmony involving body, mind, emotions, and spirit" (Social and Emotional Health section, ๆ1). It is clear from this statement that good health within the Aboriginal population must be holistic, encompassing not only the physical aspects of a person, but also the emotional aspect. Although research has shown that Aboriginal peoples are sharing a disproportionate amount of physical illness due to their weight (e.g., diabetes, cardiovascular disease), health is comprised of more than just the physical body. As has been briefly noted, body-related emotional experiences may have many implications on overall health; therefore, it is important to provide insight into these experiences.

\subsubsection{Body-related Emotional Experiences of Young Aboriginal Women}

Throughout this section, an in-depth review of the key studies that have examined young Aboriginal women's body-related emotional experiences will be provided. Although very little literature has examined the body-related emotional experiences of young Aboriginal women, the studies that do exist have tended to focus on negative experiences. Large-scale studies have indicated that levels of body dissatisfaction among young Aboriginal women are high, and as Cash (2002) has suggested, there are many dysphoric emotions (e.g., anxiety, dejection, shame, disgust) that may result when a person evaluates his or her appearance unfavorably.

With regards to Canadian Aboriginal youth, Gittelsohn et al. (1996) examined body shape perception in an Ojibway-Cree community in Northern Canada: A total of 
729 men and women, ages 10 years and older, from the Sandy Lake First Nation Band took part in this study. The highest percentage of participants was within the 10-19 year-old age range. Using a set of outline drawings, which ranged from very thin to very obese, researchers tried to determine levels of body shape satisfaction among their participants. Results indicated that $61.3 \%$ of the population desired a thinner shape, and more women than men wanted a slimmer size. Furthermore, only $16 \%$ of the population was satisfied with their current body shape, and those individuals who had a higher body mass index were less satisfied with their bodies than those individuals with a smaller body mass index.

Another Canadian study that examined the prevalence of overweight and the associated behavioural factors involved over 600 participants from the Sandy Lake First Nation Band in Northern Canada (Hanley et al., 2000). One of the main findings was that there was an inverse relationship between overweight status and the heaviness of the healthy female body image selected; a pattern not found in males. These findings indicate that being overweight might alter young Aboriginal women's perception of what is deemed a healthy weight.

Aside from the above-mentioned Canadian studies, there have also been American studies that have examined issues associated with the body. Story et al. (1994) studied over 13,000 participants via a large school-based survey that was conducted between 1988 and 1990 by the Indian Health Service. Participants were youth in grades 10-12 living on or near reserves across the United States. The purpose was to determine weight perceptions and weight control practices among American Indian-Alaska Native adolescents. Results showed that girls in grades 10-12 were more likely to report feeling overweight, dissatisfied with their body, and low body pride 
compared to those girls in grades 7-9. Furthermore, in a similar study that involved an ethnically diverse sample, Story et al. (1995) found that when compared to rural white adolescents from similar areas, American Indian females were less satisfied with their bodies

In another study, in which researchers drew a sample of over 11,000 from the survey conducted between 1988 and 1990 by the Indian Health Service, NeumarkSztainer, Story, Resnick, et al. (1997) compared the psychosocial and weight-related concerns among overweight and non-overweight Native American adolescents living on or near a reserve. They found that there were increases in low body pride, body dissatisfaction, and weight concerns with increasing overweight status.

Using a similar data set, Cummins et al. (1999) used information from over 13,000 participants to determine factors associated with physical and emotional health in a population that was at risk for poor health outcomes. They found that there was a strong positive correlation between emotional health and body pride for females. Furthermore, satisfaction with the physical-self among adolescents was one of the most important markers of self-esteem.

Aside from the survey that was commissioned by the Indian Health Service, another survey, known as the Minnesota Student Survey, was conducted in 1989. This survey, which included 92,000 students in grades 6,9 , and 12 , has been the basis of many studies that have examined body-related issues among adolescents (e.g., Gruber \& Ponton, 1995; Neumark-Sztainer, Story, French, et al., 1997; Story et al., 1995). The Minnesota Student Survey included an ethnically diverse sample and focused on perceptions of body weight and other weight related measures among adolescents of different ethnicities. In one of the studies, Story et al. (1995) examined the differences 
in dieting behaviours between American Indians, Hispanics, and whites and found that American Indian adolescents experienced one of the highest rates of intentional vomiting (i.e., weight control behaviour).

Many researchers who have been interested in examining the body-related issues that are experienced by Aboriginal youth have used data that was collected for the Indian Health Survey and the Minnesota Health Survey. However, not all researchers have used data from these surveys. For example, a recent study by Rinderknecht and Smith (2002) examined body image perceptions among 155 urban Native American youth, aged 5-18 years. Using a body image tool, which required the participants to view silhouettes, they found that $61 \%$ of girls expressed a desire to be thinner. Furthermore, those participants who expressed the greatest dissatisfaction with their body were overweight girls. Although the youth were compared across different ages, regardless of age, similar levels of body dissatisfaction were experienced.

When examining the literature on the body-related experiences of young Aboriginal women, it seems clear that the majority of the studies have found that young women are experiencing body-related concerns. However, it is important to gain a wellrounded understanding of the experiences of young Aboriginal women because it does not appear to always be negative. As indicated by the Social Sciences and Humanities Research Council of Canada (McNaughton \& Rock, 2003), we have to move away from the "epidemiological emphasis on the negative" when conducting Aboriginal research.

\subsubsection{Positive Body-related Experiences}

Many findings from previous studies suggest a negative impact of weight on body satisfaction; however, Neumark-Sztainer, Story, Resnick, et al. (1997) found that 
there are only weak associations between weight status of Native American youth and social and psychological concerns. They suggested that being overweight only has a limited impact on psychosocial health. Further, overweight American Indian girls have been shown to perceive their weight more positively than non-overweight American Indian girls (Neumark-Sztainer, Story, French, et al., 1997).

Similar findings by Thompson et al. (2002) identified American Indian women as more accepting of larger body types. Through the use of focus groups, they explored the environmental, cultural, and policy-related determinants of physical activity among sedentary American Indian women. A qualitative design was utilized because, as stated by Thompson et al., they sought an in-depth exploration of these determinants and they did not feel that this could be achieved by using a more structured survey. Participants claimed they were less active than other cultures because they did not feel pressured to lose weight and maintain a slim figure. This research team illustrated how the culture of American Indians is less competitive about physical appearance and more accepting of larger body types than the non-Hispanic white culture.

Similarly, in the Canadian study that examined weight perceptions within an Ojibway Cree community, Gittelsohn et al. (1996) found that although over half of the young women sought a thinner shape, participants identified an ideal figure that was larger than reported in other Western populations. This supports the belief that young Aboriginal women are more accepting of larger body types.

As just indicated, some researchers have tried to examine the positive attributes (i.e., acceptance of larger sizes) of young Aboriginal women; however, little research has used this as the main focal point. Nevertheless, the bulk of research that exists with regards to the body-related emotional experiences of young Aboriginal women, whether 
focusing on the positive or negative emotions, are consistent with what we know about young women in general.

\subsubsection{Consistency Among all Young Women}

As discussed, the majority of body-related literature surrounding young Aboriginal women has focused on the negative experiences; this seems to be the case for young non-Aboriginal women as well. Page (1991) found that most young women were not satisfied with their present weight in a study that involved over 600 , primarily white, adolescent females. Over half (54.6\%) of the young women rated themselves as being "too fat", whereas only $17.1 \%$ of the young women could have been classified as overweight.

Many young women are experiencing negative emotion with regards to their body and this has been of concern to many researchers. Piran (2001) noted that although adolescent women are now provided with career and social opportunities that previous generations only dreamed of, the body has remained an oppressed domain. Adolescence, that turbulent time around 12 to 16 years of age, is a time in which embarrassment often occurs and a time in which social situations or places are often avoided in attempt to protect from ridicule (James, 2000). Furthermore, throughout adolescence young people begin to make sense of who they are within the larger context of the society in which they live (Heilman, 1998). By the time adolescence is reached, many girls realize that they are overly obsessed with their appearance, yet they are at a loss as to where else they can focus their attention (Sweeney, 2001).

Adolescents may be obsessed with appearance because they internalize, consciously and unconsciously, what society views as feminine and masculine 
(Sweeney, 2001). More specifically, as young girls become women they have internalized how important it is to have a beautiful body and they have learned that the power of their gender comes from looking good, not from their personality or achievements (Sweeney, 2001). Pinto (2004) has indicated that many young women are highly critical of their bodies and are often concerned about the appearance of their physiques in many situations.

James (2000) conducted a study in which she examined a specific situation (i.e., swimming pool) in which young women may be concerned about their physiques. She explored the attitudes of girls with regards to public swimming pools. The ethnicities of the young women were not noted; however, James did indicate that it was a diverse sample that consisted of girls of varying socioeconomic status. The purpose of the study was to examine how adolescent girls felt when they were in a position of potential embarrassment or ridicule due to being in a bathing suit in front of a perceived audience. All of the girls in the study expressed an awareness of presentation of their physical-self at public swimming pools. Few girls reported feeling good about their appearance when swimming at public pools, and most girls reported feeling fat or ugly at least some of the time. James (2000) also found that young women were uncomfortable when swimming in public swimming pools because their bodies were on display in an outfit that allows for maximum appraisal and evaluation by others. These findings are consistent with Dittmar et al. (2000) who stated that the awareness of other's evaluations of self is heightened during adolescence.

When individuals feel that they are being evaluated they may engage in the process of self-presentation, in which they attempt to control the impressions that other people form of them (Leary \& Kowalski, 1995). For example, individuals who 
positively view their bodies may dress in certain ways in an attempt to accentuate the attractive features of their body (McAuley \& Burman, 1993). However, when people are motivated to present desired impressions, but have doubts about their ability to present that impression, they will experience social anxiety (Schlenker \& Leary, 1982). When an individual experiences social anxiety, in combination with physique-related concerns, they experience social physique anxiety (SPA) (Hart, Leary, \& Rejeski, 1989). Hart et al. (1989) have suggested that those who are satisfied with their bodies and not concerned with others' reactions to their physiques might rarely experience SPA. However, many individuals are continually worried about how others view their physiques, either because they are objectively unattractive or because they hold an unrealistic and negative perception of their physiques (Hart et al., 1989).

Although it is thought to be somewhat of a norm that young woman experience anxiety due to their physiques, many researchers have tried to determine why young women feel this way about their body. As mentioned, it may be because the young women have internalized, at a young age, the importance of having a beautiful body (Sweeny, 2001). Furthermore, James (2000) described how many adolescent girls may be unhappy with their physical appearance because their bodies, especially during puberty, are not matching up to the unrealistic ideals that are portrayed in the media.

Although the influence of media may have an effect on many young women, the negative implications of the media may have even more of an adverse effect on young Aboriginal women since the psychological development of these young women can be "undermined by mass media's general lack of positive representations of Native Americans" (Aidman, 1999, p. 134). Given the ideals that are portrayed in society it is not surprising that, as stated by Vasquez and Fuentes (1999), adolescence is a time when 
socializing is hindered by ethnic differences. Although it is not common to see young Aboriginal women portrayed in the media, the few Native Americans that are portrayed (i.e., Disney's Pocahontas) have also been depicted as the "ideal girl" with long legs, a tiny waist, and large breasts (Aidman, 1999). These images may be problematic to the overall well-being of adolescents because adolescence is a time when young women view their bodies as the culture views them (Pinto, 2004).

Similar to many researchers who have been interested in examining why (e.g., media) so many young women experience high levels of body dissatisfaction, Rand and Resnick (2000) tried to determine if young women were in fact dissatisfied with their body. This research, which included primarily white participants, involved the reexamination of the measure of body size dissatisfaction. The pair thought that it was important to try to avoid the bias toward negativity (i.e., body dissatisfaction) by reexamining what is actually a "good enough" body size. They proposed that individuals could consider their body size to be "good enough", and socially acceptable, even though their actual body size may not match their ideal. Similar to other studies that included measures of body size dissatisfaction (e.g., Gittelshon et al., 1996; Story et al., 1994), participants in this study were shown an array of line drawings. These drawings were consistent with their age range and gender, and the participants were asked to select the body size that resembled how they currently look and the size that reflects how they would most like to resemble. However, different from other studies, Rand and Resnick also asked the participants to select the body size that was "socially acceptable". More specifically, the participants were asked, "When you see people at school, at the mall, or on the beach, what body sizes do you think look okay? Circle the number under all the body sizes you think are acceptable in addition to the size you like best" (Rand \& 
Resnick, 2000, p. 310). Body size acceptability occurred when the researchers found that a subject's actual size matched a body size that was identified by the participant as acceptable.

The results of Rand and Resnick (2000) indicated that a large majority (87\%) of participants considered their body size to be socially acceptable even though it may not have matched their ideal; this occurred for both males and females. When examining adolescents alone, it was found that $92 \%$ of normal weight and $84 \%$ of overweight adolescents viewed their body size as acceptable. Even for obese adolescents, although 91\% rated their current body size differently from their ideal size (i.e., what previous research calls body size dissatisfaction), it was found that almost half (47\%) still viewed their body size as socially acceptable.

Rand and Resnick (2000) argued that the discrepancy in actual size to ideal size should not be assumed to mean body size dissatisfaction; however, there were limitations in their study. First, participants in this study, and in studies using similar line drawings, made evaluations on body sizes that are portrayed in swimsuits (i.e., apparel that maximizes critical appraisals). The results of the study may have been different if the participants evaluated body sizes in different apparel. Second, Rand and Resnick indicated that their sample was limited in its representation of all adolescents and contexts. Further research is needed to determine whether or not acceptability is the same when applied to others, and in different contexts.

Even though Rand and Resnick's (2000) study has its limitations, it has made great strides in highlighting the possible positive attitudes (i.e., social acceptability) associated with the body as opposed to focusing purely on the negatives (i.e., body size dissatisfaction). Wright (1991) has stated that many researchers have a tendency to 
focus on negative feelings and attributes, and concerns surrounding the body are commonly referred to as normative discontent and women's "problem" instead of a possible problem with theoretical and methodological approaches (Bayer \& Malone, 1996). Although previous studies have been warranted in the exploration of body size dissatisfaction, Rand and Resnick noted the limitations associated with body size dissatisfaction research. They stated the importance of expanding the literature into the area of body size acceptability instead of focusing on body size dissatisfaction, which may restrict the view of what is acceptable.

\subsubsection{Limitations of Previous Research With Young Aboriginal Women}

Similar to the limitations that accompany the studies that have examined young non-Aboriginal women's body-related emotion, literature involving young Aboriginal women is also limited. Although there have been quantitative studies conducted that have examined body-related concerns among Native Canadian (e.g., Gittelsohn et al., 1996; Hanley et al., 2000) and Native American (e.g., Cummins et al., 1999; Gruber \& Ponton, 1995; Neumark-Sztainer, Story, Resnick, et al., 1997; Rinderknecht \& Smith, 2002; Story et al., 1994) adolescents, there have been limited, if any, qualitative studies that have examined the body-related emotional experiences of young Aboriginal women. The researchers who conducted these previous quantitative studies were working within positivist and post-positivist paradigms. These paradigms generally work from within realist ontology and objective epistemologies, often relying on surveys, experimental, and quasi-experimental methodologies (Lincoln \& Guba, 2000). Seeing as the research within these paradigms is often quantitative inquiry, the information collected is limited by the questions asked. In addition, these researchers are often concerned with 
prediction, objectivity, replicability, and generalizations. Although this quantitative research is important, researchers need to try to understand the experiences of young Aboriginal women in different ways (i.e., qualitative research).

Qualitative research can help to provide an in-depth understanding of young Aboriginal women's body-related emotion in a number of different ways. First, and somewhat different to traditional quantitative research, Creswell (1998) has indicated that qualitative researchers are the instruments of data collection and that the research is undertaken in natural settings. Second, he explained how numerous methods of data collection (e.g., focus groups, one-on-one interviews) are used to develop a holistic picture of the complexities surrounding social or human problems. Furthermore, the multiple perspectives of participants can be illuminated through the use of semistructured interviews involving open-ended questions (Patton, 2002).

In addition to the lack of research utilizing qualitative methods when studying the body-related emotions of young Aboriginal women, there are a number of other methodological limitations that must be addressed. First, the majority of the large-scale studies that have examined the body-related concerns among young Aboriginal women have been with those adolescents who live on or near a reserve (Gittelsohn et al., 1996; Neumark-Sztainer, Story, Resnick, et al., 1997; Story et al., 1994). Therefore, the research is limited in that we have only been provided with a restricted view of the experiences of young Aboriginal women because the experiences of urban young women have not been shared. The importance of including urban youth in future research has been noted by the Report of the Royal Commission on Aboriginal Peoples (RCAP; 1996). The RCAP has indicated that almost half of Canada's Aboriginal youth population lives in non-reserve urban centers and in order to understand the situation of 
Aboriginal youth, it is critical to understand the urban context in which many of them live.

Second, aside from the fact that many of the studies that have examined bodyrelated concerns among Aboriginal youth only included those individuals who live on or near a reserve, it is also important to note that most of the large-scale studies have only examined American Aboriginal adolescents (e.g., Cummins et al., 1999; NeumarkSztainer, Story, Resnick, et al., 1997; Story et al., 1994). Canada's Aboriginal youth population (those 15 - 24 years old) includes over 400,000 people (RCAP, 1996) and Canadian Aboriginal youth make up 56.2 per cent of Canada's Aboriginal population (RCAP, 1996). Although Canada has a large number of Aboriginal youth, they have not been provided the opportunity to share their body-related emotional experiences within the formal academic literature. Although many of the American studies have been helpful in identifying possible issues (e.g., negative emotions surrounding the body) that should be addressed within this particular study, it is important to realize that the participants of this study will be Canadian residents, and therefore, experience a different cultural context.

Third, although the sample of participants is a limiting factor of many studies, it should also be noted that much of the literature on body-related concerns experienced by Aboriginal adolescents (e.g., Cummins et al., 1999; Neumark-Sztainer, Story, Resnick, et al., 1997; Story et al., 1994) is derived from a single survey that was conducted between 1988-1990 with American Indian and Alaska Native youths in grades 7-12. This comprehensive survey involved more than 15,000 Native American youth and has been helpful in identifying some of the emotions (i.e., anxiety; pride) that are experienced by young Aboriginal women with regards to their bodies. Nevertheless, 
much of the literature that was produced is limited in the fact that they were based on just one survey that was conducted fifteen years ago. The body-related emotional experiences of young women today might be quite different to the experiences of young women from over a decade ago. For example, more explicit images of women are now being portrayed in the media (e.g., Spice Girls) either as a result of a change in women's attitude (e.g., "girl power") or a media portrayed movement (Gamble, 2000). Therefore, these media portrayed images may be impacting the experiences of today's young women.

Lastly, the large-scale survey involved self-report measures of height and weight, which were then used to calculate BMI and used to determine whether or not the participants were normal weight, overweight, or obese. However, Himes and Story (1992) have shown that American Indian youth, ages 12-19 years, have a tendency to significantly underreport weight, thereby altering the BMI values. Furthermore, the survey was administered in a school setting, which Page (1991) indicated could be a limitation since adolescent females may not provide accurate measures when the information is reported in a classroom setting. The inaccuracy may be a result of social desirability bias, or simply due to the fact that respondents do not accurately know their height and weight measures (Page, 1991). Self-report measures of BMI are a limitation to the studies because many of the researchers have classified their participants as overweight, when in fact they may not be.

Many of the methodological limitations that have been addressed have helped to guide this study in a way that will help to provide insight into the body-related emotional experiences of young Aboriginal women. Quantitative research has indicated that dissatisfaction with the body and negative body image are experienced by young 
Aboriginal women (Gittelsohn et al., 1996; Neumark-Sztainer, Story, Resnick, et al., 1997; Story et al., 1994). Nevertheless, there have been little, if any, qualitative studies that have tried to gain an in-depth understanding of these body-related emotional experiences. It is possible that these young women may experience many positive emotions (e.g., body pride), but much of the previous research has not examined this possibility. As a result, this research has attempted to fill this important gap in the literature.

\subsubsection{Feminist Perspective}

Feminist theory was used as an overarching perspective for this study. Feminism is a theoretical paradigm and an ideological perspective that is used to draw attention to the needs of people and enables researchers to lift the voices of marginalized people (Creswell, 1998; Denzin \& Lincoln, 2000). Feminist research differs from traditional research in three unique ways. First, feminist research is politically motivated. It is grounded by a political movement that challenged women's subordination and is derived from changes in women's experiences and situation (Alway, 1995). Second, feminist research begins with the experiences of women. It is a voice-centered approach to research based on women's experiences and listening to their stories (Way, 2001). Feminism is a complex perspective having no single feminist voice (Alway, 1995). This is an important concept to keep at hand because the intent of this research was to gain a holistic understanding of the emotional experiences of young Aboriginal women, and feminist theory has allowed me the chance to tell the unique stories of each individual as well as their collective story. Third, feminist researchers try to alleviate the power imbalances between the researcher and researched that are so common within traditional 
research. One method of demonstrating how the power imbalances have been reduced in this study is by using first-person voice throughout the remainder of this thesis. Denzin (2000) has described how some writers will make themselves present within the text through the use of first-person voice in order to provide in-depth accounts of everyday life. He further explained how this presence within the text often portrays the writer as a participant and therefore the traditional hierarchical approach that is often taken by the researcher has been lessened.

Feminist researchers work with women to try and understand how women's lives are "contextualized" and "framed" (Olesen, 2000). A feminist approach encourages multiple encounters with women so that their experiences are told in their own terms. Alway (1995) has stated that feminist research can be used to "produce less false, less partial, and less perverse representations without making any claims about what is absolutely and always true" (p.225). Once again, by allowing each of the young women in this study to express their own ideas and experiences I had hoped to gather information that represented their lives as accurately as possible.

As previously mentioned, positivist and post-positivist paradigms have typically framed the research that has been conducted with regards to the body-related emotional experiences of young Aboriginal women. Stein (1997) has stated that research progress on the health of women has been hindered by the limitations of the dominant positivist method that is often undertaken within the field. She wrote how the constricting nature of the positivist framework may not support the broad investigation in women's health. I do not fully agree with Stein's statements when she described positivist research as hindering; positivist research also has its strengths (i.e., often has large sample sizes). However, I do not feel that a solely positivist framework could provide an in-depth 
understanding of the experiences of young Aboriginal women. When examining the paradigms within which researchers work, I realize that the boundaries between the paradigms remain "fuzzy" (Patton, 2002). Nevertheless, previous research has dictated a path for me by illuminating the lack of descriptive research that is available with regards to this particular issue. Therefore, I felt that a study derived from a feminist paradigm could add to the current body of literature.

Feminism was an appropriate perspective for this particular study because it "has a special role in supporting young women's voicing of problematic experiences in the body domain" (Piran, 2001, p. 172). Piran also stated that young women play a major role in enhancing feminist theory and development. My intent was to have young women play an integral role in this study because they are the ones who would be rendering the information to help educate others. The concept of the "universalized woman" is fading (Olesen, 2000), and it was important that I hear the voice of each young woman. All individuals experience things differently and it is important to hear their individual stories. "To speak is not sufficient; to be heard is critical"(Pinto, 2004, p. 81). Vasquez and Fuentes (1999) has said that an inadequate amount of research has been conducted on the unique lives of non-white young women and thus it was important to hear the stories of young Aboriginal women. In addition, it was important for me to recognize that although female subordination is collective, it was also important to understand each individual experience, as best as possible, within her cultural context. Reinharz (1992) has indicated that some feminist researchers choose not to mention their feminism due to the prejudice associated with the term. However, I felt that it was important to make this distinction. I would have been providing the participants injustice by not acknowledging their unique experiences as women. 
Although I felt that it was important to listen to and represent the stories of young Aboriginal women, Gillies and Alldred (2002) have indicated that some feminist researchers do not feel that researchers should speak for others. They pointed out that researchers should be limited to representations of themselves and their immediate communities; this remains an ongoing debate and dispute. My position on whether or not I, as a white feminist researcher, should study the stories of young Aboriginal women was summed up best by Edwards (1996); "can I possibly be justified in leaving them out?" (p. 83).

Now that feminist theory has been discussed, it is important to step back and examine the ontological and epistemological beliefs that are held by many feminist researchers. With regards to the ontological premise, feminist researchers often believe that multiple realities exist, including the realities of the researcher, the researched, and the reader interpreting the study (Creswell, 1998). In terms of reality, many feminist researchers also believe that a woman's real world is influenced by race, class, and gender (Collins, 1986; Denzin \& Lincoln, 2000). Therefore, a young woman's race, class, and gender will influence her perception of reality. It was important to remember this when developing the interview guides that would be used to try to understand the realities of the young women. Although it has been stated that feminist researchers believe in multiple realities, it should also be noted that the ontological beliefs of feminist researchers can differ in terms of the objects being researched and the nature of reality (Ramazanoglu \& Holland, 2002).

In addition to ontological beliefs, the epistemological premise of feminist researchers is that they try to minimize the distance between the researcher and the researched. However, since there are numerous epistemological standpoints, as there are 
ontological beliefs, feminists can draw on differing epistemologies. Reinharz (1992) describes how many researchers within a feminist paradigm often note a sense of connectedness between the researcher and the researched. Researchers, whose beliefs fit within the feminist paradigm, often realize that the absolute objectiveness, which is sought by pure positivists, is impossible to attain (Patton, 2002). Nevertheless, Patton (2002) described how feminist researchers still strive for objectivity, while admitting that they are working within a "methodologically imperfect world" (p.93). In recognition of this epistemological belief I knew that it was important to use multiple methods to try to provide as much insight as possible into the emotions experienced by the young women. Nevertheless, it was recognized that absolute objectiveness would not be obtained.

This research project used feminist theory as a guiding perspective for qualitative inquiry. The ontological and epistemological beliefs of feminist researchers are consistent with qualitative research. Baumgartner and Strong (1998) described how attractive qualitative methods could be to feminist researchers because qualitative researchers are concerned with experience, which is parallel to feminists who are interested in women's experiences. Figueira-McDonough (1998) described how qualitative methods can be an effective tool to address questions and/or populations for which there has been limited prior inquiry. Creswell (1998) described qualitative inquiry as a "process of understanding based on distinct methodological traditions of inquiry that explore a social or human problem. The researcher builds a complex, holistic picture, analyzes words, reports detailed views of informants, and conducts the study in a natural setting"(p. 15). Since there has been very little research that has tried to 
understand young Aboriginal women's body-related emotional experiences, I felt that qualitative inquiry could provide young women with the opportunity to tell their stories.

The above-mentioned definition of qualitative research was chosen because it emphasizes a holistic and complex picture of the issue being investigated (Creswell, 1998). This definition encourages qualitative researchers to examine the various dimensions of an issue and display its complexities. When trying to provide insight into the body-related emotional experiences of young Aboriginal women, it was important to examine the various dimensions and then, as accurately as possible, display these complex relationships. Issues surrounding the body can be very complex, and this form of research allows researchers to investigate topics in all their complexity (Bogdan \& Biklen, 1998). Although quantitative studies have been useful in identifying how psychosocial variables such as global self-esteem, body esteem, and body dissatisfaction are related to social evaluation of the body (Eklund \& Crawford, 1994), Patton (2002) stated how the questions in quantitative studies are standardized and therefore the responses are limited to predetermined categories. Allison (1988) also described how quantitative research has been inefficient in its attempt to provide insight into the complexity of women's attitudes and practices, especially when working with marginalized groups such as minority women. Even though there is some overlap between the two types of inquiry, qualitative research differs from quantitative research in many aspects.

The word qualitative implies an emphasis on meanings and process that are not thoroughly examined in terms of quantity, amount, intensity, or frequency (Denzin \& Lincoln, 1994). Qualitative researchers are often concerned with the socially constructed nature of reality, the personal relationship between the researcher and what 
is studied, and the situational limitations that shape inquiry. In addition, qualitative researchers generally try to seek answers to questions that stress how social experience is created. Quantitative studies, on the other hand, typically stress the measurement and analysis of causal relationships between variables, not processes (Denzin \& Lincoln, 1994).

\subsubsection{Conceptual Framework}

Although feminist theory was used as the guiding perspective for this particular study, Cognitive-Motivational-Relational Theory (Lazarus, 1991) was utilized as the conceptual framework. Creswell (1998) described how theory could be used multiple ways within qualitative research, and Yin (1994) also explained how theory could be used to guide a study in an explanatory way. The theory was used as a framework for understanding the body-related emotions experienced by young Aboriginal women. More specifically the theory was used as a guideline when developing interview guides and when interpreting the data.

Cognitive-Motivational-Relational Theory explains how emotions emerge from complex interactions between a person, behaviour, and the environment (Lazarus, 1991, 2000). The theory is "relational" in that emotions involve person-environment relationships; the person and environment are not viewed as separate units (Lazarus, 1991). The theory is "motivational" because emotions are reactions to the active goals that individuals set in their everyday lives (Lazarus, 2000). We only react emotionally when we have goals set for the outcome of an encounter. Lastly, the theory is "cognitive" because individuals are constantly making decisions about the significance of particular encounters with the environment (Lazarus, 2000). Emotions have been 
described as complex psychophysiological states that have adaptive functions and part of an ever-changing process due to a constant shift in the person-environment relationship (Lazarus, 1991). The emotional process is very complex in that there are many different emotions (e.g., joy hope, anger) that a person can experience in a person-environment relationship (Lazarus, 1991). Lazarus (1991) has provided a detailed list of the many different emotions that can be experienced (e.g., anxiety, pride).

Since this theory recognizes the complexity of the emotion process, I felt that this theory would help to provide insight into the body-related emotional experiences of young Aboriginal women. Lazarus explained how each emotion has its own natural action tendency, which can be altered by coping. For example, the action tendency of fear is avoidance or escape but an individual can counteract that natural tendency in most situations. Emotions also have their own pattern of physiological change, which is partially a result of the action tendency (Lazarus, 2000).

Appraisal and coping are the two key components of emotion (Lazarus, 1991). It is critical to understand the process of appraisal and coping when trying to regulate emotion and the subsequent consequences (Crocker, Kowalski, Graham, \& Kowalski, 2002). Crocker et al. (2002) stated that it is important to understand that the relationship between appraisal, coping, and emotion is bi-directional; it is a constantly changing process. They explained how the process of appraisal generates an emotion, which is linked to characteristics of physiological changes, cognition, and subjective feeling. Individuals appraise situations based on what is at stake due to the outcome of a certain encounter (i.e., primary appraisal) and based on what can be done in a specific situation (i.e., secondary appraisal) (Lazarus, 2000). For example, a young woman may appraise a situation as threatening because she believes that others will not like her because of the 
way she looks (i.e., primary appraisal) and that there is nothing she can do about it (i.e., secondary appraisal).

The individual must then cope with the emotions that are experienced. Coping can be achieved by problem-focused coping (i.e., directly changing the situation) or by emotion-focused coping (i.e., directly handling the emotion) (Lazarus, 2000). For example, a young woman may try to change the way she looks (i.e., problem-focused coping) and/or tell herself that the others who are evaluating her are not as pretty as her (i.e., emotion-focused coping); the strategy that an individual chooses depends on the specific situational context.

Consistent with Cognitive-Motivational-Relational Theory, Walters and Simoni (2002) proposed a model that demonstrates how cultural buffers could help with the coping process. Their "Indigenist" Stress-Coping model articulates how stress and coping processes operate within the fourth world context of Native women. The fourth world context is the situational existence of a minority indigenous population within a nation in which a colonizing and subordinating majority holds privilege and power (O'Neil, 1986). This model depicts how within an indigenous population, the health outcomes (i.e., physiological, emotional, psychological) that are experienced are the results of different stressors (i.e., trauma); however, the effects of stressors are moderated by cultural buffers (e.g., identity attitudes, spiritual coping) which help in coping. Therefore, while still working within Cognitive-Motivational-Relational Theory, it was important to recognize the role of cultural buffers throughout the coping process.

When working within the proposed framework, it was also important to continuously identify the "fourth world context" while working with the young women and talking about body-related emotional experiences. As noted by Walters and Simoni 
(2002), the behavioural health problems that are experienced by Native women can be connected to their colonized status, and associated with the many forms of discrimination. Although other researchers (e.g., Kirmayer et al., 2000) also support the notion that colonization has lead to numerous mental and physical health consequences within the Aboriginal population, few studies have explored the effects of colonization on Aboriginal peoples health. Therefore, it was important within this study to recognize the impact of colonization and fourth world context so that I would not miss out on important aspects of the young women's experiences and stories.

Ultimately, Cognitive-Motivational-Relational Theory was chosen as the conceptual framework for this study because of its emphasis on the need to understand the role of appraisal and coping in emotional experiences. Consideration of these processes within the fourth world context, as well as cultural buffers, was also noted throughout the study.

\subsection{STATEMENT OF THE PURPOSE}

The purpose of this qualitative case study was to provide insight into the bodyrelated emotional experiences of young Aboriginal women. Young Aboriginal women are experiencing many issues related to their bodies (Neumark-Sztainer, Story, French, et al., 1997; Neumark-Sztainer, Story, Resnick, et al., 1997; Rosen et al., 1988; Story et al., 1995; Story et al., 1994). Although many young women are concerned about the appearance of their physiques in various situations, young Aboriginal women may be of increased concern because they tend to be more overweight than white individuals (Story et al., 1995) and experience more mental health problems than their peers (MacMillan et al., 1996). However, there are many emotions that a person can 
experience related to the body, and it was important to gain a well-rounded understanding of the experiences of young Aboriginal women because they do not appear to always be negative (Neumark, Story, French, et al., 1997).

Feminist theory was used as an overarching perspective for this study. This theory recognizes the importance of listening to women's stories and hearing women's experiences (Way, 2001). Cognitive-Motivational-Relational Theory was used as the conceptual framework because it was the emotional experiences that I was interested in, and this theory recognizes emotion as a complex and dynamic process (Lazarus, 1991). 


\section{CHAPTER 2}

\subsection{METHODOLOGY}

Having decided upon feminist theory as the guiding perspective, and CognitiveMotivational-Relational Theory as the conceptual framework, for providing insight into the body-related emotional experiences of young Aboriginal women, it was important to decide upon the strategy that could provide insight into the body-related emotional experiences of young Aboriginal women. First, I will describe why a qualitative case study was an appropriate strategy of inquiry. I will then describe the methods that were used to provide insight into the complex nature of the body-related emotional experiences of young Aboriginal women. Lastly, I will provide a detailed description of the process used to analyze the data.

\subsubsection{Case Study Approach}

The qualitative method of inquiry that was chosen for this study was case study. Maintaining a feminist perspective, while utilizing case study methods, helped to keep the study focused on what was important; women's experiences. Stake (1995) identified a case study as "the study of the particularity and complexity of a single case, coming to understand its activity within important circumstances" (p. xi). Therefore, a case study approach was used to help keep the study focused on catching the complexities of the

case being investigated (Stake, 1995). Creswell (1998) identified a case study as the 
investigation of a "bounded system" over time by the use of detailed data collection. The "bounded system" is the case that is examined and it is bounded by time and place.

Stake (1995) indicated that people are often cases of interest and that we are interested in them not only for their uniqueness, but also for their commonality. Some feminist researchers (Reinharz, 1992) have stated that the power of a case study comes from its fundamental ability to clearly convey the dimensions of individual life or social phenomenon. Although it is possible to study a single individual, Reinharz (1992) has suggested that the study of groups of women is more common in feminist research than the study of individual women. Each young woman was a case of interest for this particular study, and each case was instrumental to learning about this particular group and their body-related emotional experiences.

Case studies can be sorted into three different categories (i.e., intrinsic, instrumental, collective), depending on the type of case that is chosen for the study (Stake, 1995). Stake (1995) described how those researchers who are interested in the case itself would engage in intrinsic case study, whereas those researchers who are interested in providing insight into a particular issue would engage in instrumental case study. With regards to instrumental case study, the researcher will often choose a case based on its ability to provide insight into the issue of interest. A collective case study can be considered an instrumental case study that is extended to several cases. Therefore, a number of cases may be examined in an attempt to provide insight into a particular issue. Within this study, a small group of young Aboriginal women helped to provide insight into the body-related emotional experiences of young Aboriginal women; therefore, this study was a collective case study. In collective case study there is an important co-ordination between the individual cases (Stake, 1995), and this was 
important because I was interested in similarities and differences across, and within, each case.

\subsubsection{Data Collection}

Researchers conducting case studies often use a wide variety of data collection methods to construct an in-depth view of the case (Creswell, 1998). Creswell (1998) stated that the data collection within case studies is extensive, consisting of various sources of information. This study utilized interviews, observations, field notes, and art elicitation as the main methods of data collection. Interviews were the principle method of data collection. Interviews, as described by Stake (1995), are one of the main avenues for the exploration of multiple realities. Observations were also an important component in data collection, as were field notes. Bogdan and Biklen (1998) described how researchers often record field notes so that there is a written account of what he/she sees, experiences, hears, and thinks throughout the data collection and reflection process.

Data was collected over five phases (i.e. participant selection, rapport building, focus group, one-on-one interviews, art project). Prior to phase one I positioned myself as the researcher (i.e., told my personal story) so that the perspective I was bringing to the study could be highlighted (Stake, 1995). Creswell (1998) described how researchers can position themselves by disclosing personal values, biases, and contexts that may shape the research. Also, prior to phase one, a brief overview of the special considerations that were taken throughout the data collection has been provided.

Phase one was the participant selection phase in which I was introduced to a known sponsor who subsequently introduced me to a group of young women who would in turn become participants for this study (see section 2.1.2.3). The second phase of the 
study was a rapport building phase in which the participants and I spent time getting to know one another better (see section 2.1 .2 .4 ). This time was a chance for me to try to gain the trust of the participants. Phase three involved a focus group session (see section 2.1.2.5). The young women reacted to pictures of women depicted in popular fashion magazines and discussed these images. The young women then developed collages from pictures taken from fashion magazines. The goal of this phase was to have the young women develop collages that represented how they felt about their own bodies so that discussion could be generated about issues surrounding the body. Phase four involved one-on-one interviews in which each of the young women met with me separately to discuss issues surrounding their body and issues faced by young Aboriginal women in general (see section 2.1.2.6). Lastly, phase five involved the development of art projects by the participants (see section 2.1.2.7). The participants were provided the opportunity to express the experiences of young Aboriginal women through the use of multiple forms of art. Although the study was organized into separate phases, it is important to note that each phase built upon one another, and no phase was by any means exclusive. The phases should be viewed as integrated units that steadily flow from one phase to another.

This multi-phase approach was used because feminist researchers should combine methods in an attempt to "illuminate previously unexamined or misunderstood experiences" (Reinharz, 1992, p.197). Morawski (1990) stated that feminists should not only listen to the experiences of women but we must also try to focus on how the experiences emerged. Since the young women that participated in the study may not have felt comfortable sharing their experiences and stories in the same manner, it was important to include multiple methods of data collection. The combination of methods 
allowed me to show respect for individual differences in the sharing of information (Reinharz, 1992).

Although a combination of methods was utilized, interviews were one of the main methods of data collection. Creswell (1998) has identified interviews, whether with individuals or groups, as a key component to all qualitative research. Multiple interviews were conducted within this study (i.e., phase 3, 4, and 5) because some feminist researchers (i.e., Rienharz, 1992) believe that multiple interviews are more accurate than single interviews. Furthermore, multiple interviews allow for feedback and further discussion of previously stated information. By conducting multiple interviews, it was also possible to ask the participants to clarify and expand upon comments that were made in previous discussions. The multiple interviews also helped to develop what I believed to be a trusting relationship between the participants and myself. Reinharz (1992) supported this notion by stating that multiple interviews are characteristic of feminist research because they help to form strong relationships between the interviewer and the interviewee.

\subsubsection{Personal Story}

Stake (1995) explained how there are many different case researcher roles (i.e., researcher as evaluator, researcher as interpreter), and it is important to position yourself (i.e., tell your story) so that the readers understand the experiences that you are bringing to the study. Since I was playing the role of the researcher as interpreter, I thought it was especially important to position myself so that I could highlight possible strengths and/or biases that I may have brought to the study. 
Since the focus of this study involves emotions that surround the body, I felt that it was important to share my own feelings surrounding my body. As a young girl and adolescent, I was always very tall and skinny compared to other female youth my age. Regardless, I still experienced concerns surrounding my body. I found it frustrating as an adolescent when others could not understand my concerns surrounding my thinness. I remember having body anxiety because I could not buy certain clothes because my body did not fill them out. Also, I spent every summer at a local lake because I was involved in sprint kayaking and canoeing, and I remember not wanting to wear twopiece bathing suits because I felt that my ribs stuck out. To this day, I still feel that society holds a common perception that equates thinness with body satisfaction. Now, as a woman in her mid-twenties, I am proud of my body. Although I recognize that my body is not what I perceive to be perfect, I rarely experience body-related concerns. Nevertheless, as a woman living in a society where so much emphasis is placed on the body, I am aware of the body-related issues that many individuals, especially adolescent females, face.

With regards to my research journey, it began at Dalhousie University, in Nova Scotia, where I completed my Bachelor of Science in Kinesiology. I have always had a strong interest in academics; therefore, after my undergraduate degree I moved to Saskatoon and entered a master's program. Since I have been in this master's program my research interest has been in the area of young women and their body-related concerns. Although my research interests were originally quite general, my interests soon focused on the experiences of young Aboriginal women. I feel that my interests focused because my cousins identify themselves as Aboriginal and also because Saskatoon has a large Aboriginal youth population. 
I was originally apprehensive about working with young Aboriginal women because I identify myself as white and I feared that the young women may not want to share their experiences with someone who is non-Aboriginal. However, I realized that I was able to bring a lot of strengths to a study that involved young Aboriginal women. Although it is not something that I have spent much time thinking about, I was the only female child in my family who identified as white. My adopted sister, who has been part of my family since I was just a year old, identifies herself as Egyptian. Also, my two adolescent female cousins identify themselves as G'witchen Indians. When I began to research the issues young women have with their bodies, I found it interesting that the literature has involved primarily white adolescents. In my world not everyone is white and I was determined to try to understand those experiences of non-white young women. Furthermore, I was interested in trying to understand why young Aboriginal women were supposedly experiencing more concerns with their bodies than young white women.

I feel that growing up in a family that was ethnically diverse has allowed me to become a researcher who is sensitive about issues surrounding individual identities. I have strong relationships with my family members regardless of how they identify themselves; I was raised in a family where identity was never an issue. I feel that my family instilled a sense of respect, and sensitivity, towards those people who identify themselves differently from myself. The perspective that I bring to this current study has been influenced by the experiences I have had within my family. I feel that my experiences have helped me to be understanding and compassionate when I am working with other young women who have backgrounds that are different from my own. I recognize, respect, and am intrigued by the diversity of all women. 


\subsubsection{Special Considerations}

There are many factors that I considered when I decided to work with young Aboriginal women. Smith (1999) stated that research should not be viewed as innocent exercise, but instead as an activity in which something is at stake. The knowledge of the young women was at stake and it was important for me to respect this knowledge throughout the whole research process. Smith also indicated how important it is to actively involve the participants in the creation of knowledge. In order to be more responsive and responsible to the knowledge of the participants, it is important for health researchers to have the direct input of the participants in the development of research projects (Battiste \& Henderson, 2000). By asking the participants for their input during phase two of the research I was better able to understand the perspective of the participants. Furthermore, after the data analysis, I discussed with the participants the proposed themes so that I felt comfortable in using these themes as representations of my participants' realities.

Smith (1999) stated that many Aboriginal people are hesitant about research because research has lead to policies that have intruded into every aspect of their lives. However, I made it clear to the participants in this study that the intent of this research was not to implement policy, but instead to provide insight into the body-related emotional experiences of young Aboriginal women.

In addition, The Royal Commission On Aboriginal Peoples (1996) has provided ethical guidelines for conducting research on Aboriginal peoples, which were followed. These guidelines characterize the standard of "best practice" that are accepted by the Commission (RCAP, 1996). The guidelines included such things as the importance of 
gaining informed consent, informing the participants about the purpose of the study and the expected benefits and/or risks, identifying what levels of confidentiality can be assured, and informing the participants that they will not be pressured into participation. It was important for me as a researcher to follow these guidelines so that the appropriate respect was given to the cultures, knowledge, languages, and values of the participants.

\subsubsection{Phase One: Participant Selection}

Four young women, one who was 14-years-old and three who were 18-years-old, took part in this study. The participants were recruited through a "known sponsor", which was identified as a person with a legitimate relationship with the particular group of interest (Patton, 2002). The known sponsor approach has been identified as one of the best methods of gaining entry into a particular field (Patton, 2002). After ethical approval had been obtained from the University of Saskatchewan Behavioural Research Ethics Board (see Appendix A), I organized the first meeting with the known sponsor. She was identified from my social network because of her strong ties with young Aboriginal women. The known sponsor identified herself as white and described her relationship with the participants. Informally, and also within one formal interview session, I discussed with the known sponsor her insight on the body-related emotional experiences of young Aboriginal women and the possible key issues that she felt should be addressed throughout the study. Because she is a woman who already had credibility with the participants of the study, it was important to hear her perspective on the research issue.

I also approached the known sponsor with a proposed list of criterion for the

participants. Originally, I had proposed that the participants be young Aboriginal 
women, between the ages of 14-18 years, who live in an urban center. Although large sample sizes are used in quantitative research, qualitative research only requires small samples because the credibility, meaningfulness, and insights are gathered from the information richness of the participants (Patton, 2002). Creswell (1998) indicated that case studies utilizing multiple individuals should include a maximum of 3 to 4 participants since the strength of this research comes from the depth of the information obtained from each individual, not from the breadth of individuals involved.

The term Aboriginal was chosen as the term to select the young women because it encompasses legal or status Indian, non-status Indian, Inuit, and Metis (Frideres \& Gadacz, 2001). The specified age of the participants was based on one of the largest studies that has ever been conducted on Native American youths living on or near a reserve (Story et al., 1994), which showed that females in grades 10 through 12 were more worried about their weight and their appearance compared to females in grades 79. Because I did not want to initially limit this study only to young women who attend school, I chose an age range which best represented grades 10 through 12.

The young women that took part in this study were from an urban center. This criteria was chosen because as previously stated, almost half of Canada's Aboriginal youth population lives in non-reserve urban centers (RCAP, 1996). The point of this study was not to be discriminatory to those individuals who live on a reserve, but rather for me to be able to understand the participants as best as possible from within their cultural context. I have experience living in an urban center and I felt that I was better able to understand those young women who live in a similar urban area. Furthermore, the purpose of this study was not to generalize the findings to all individuals who identify themselves as Aboriginal but, as Patton (2002) suggested, to understand the 
wide range of experiences for a small number of people. It is with the intent of this study that future researchers who read this study will be able to decide on the transferability of relevant information to other individuals who identify themselves as Aboriginal.

With the proposed criterion in mind, the known sponsor discussed with me the possibility of working with a group of young women who were part of a mentorship program at a local high school. I realized that working with this group was a unique experience that not every researcher would be fortunate enough to have. I entered an intact group who had already been meeting once a week to discuss important topics of interest (e.g., the role of media in young women's lives). Therefore, not only did I enter a group that was already comfortable speaking with one another, but I was also able to meet with them during some of their scheduled meeting times. This meant that it was not as difficult trying to organize a time that was convenient to all participants. Since this study often required a close interaction of all participants, it was important to include individuals who felt comfortable interacting with one another so that the young women could build upon one another's ideas (Esterberg, 2002). As discussed, the comfort level that is needed when speaking about sensitive issues was already established among the young women and the known sponsor felt that they would readily accept me into their group.

Once I had gained what I perceived to be the trust of the known sponsor, she introduced me to a young woman in the group (key informant) who she felt could introduce me to other group members. Key informants are individuals who are valuable in understanding what issues are occurring and why (Patton, 2002). This particular key informant offered me useful insights into the group (e.g., how often they met, 
information about each young women) and helped to identify key issues surrounding the body-related emotional experiences of young Aboriginal women. The key informant that was selected identified herself as Aboriginal. She then introduced me to the rest of the mentorship group.

When I entered this group, the young women had already been meeting for about six months. They met once a week during lunch hour and they spoke about issues that they felt were important. For example, the young women spoke about media and it's influence on young women, they also spoke about issues surrounding drug use, and any other issues that they felt were important to discuss. In addition to the weekly meetings, the young women also spent time together outside of the mentorship group. For example, the mentorship group organized an art show to raise money for a local nonprofit organization and therefore the young women spent many hours together outside of their weekly meetings.

\subsection{Group Profile}

The known sponsor, along with another woman, organized the mentorship group that consisted of grade 9 and 12 young women. The known sponsor "hand-picked" the young women in grade 12 who she felt would make "great mentors". She picked young women who had dealt with a lot of adversity in their life (e.g., drug addiction, quitting school), who had overcome this adversity, and who could mentor younger women facing similar problems. The young women from grade 9 came on a voluntary basis; many of them looking for support. Because this is a school-based program, the known sponsor formed what she believed to be a diverse group that included Aboriginal and nonAboriginal women; however, many young Aboriginal women participated. 
In addition to the key informant, three other young women volunteered to take part in the study. This group of young women all brought different perspectives to the study. Including the key informant, three of the young women (14-year-old and two 18year-olds) identified themselves as Aboriginal and one young woman identified herself as non-Aboriginal (18-years-old). All four of the participants had many, what the known sponsor called, "life stories". For example, two of the young women had previously dropped out of school but had enrolled again. Also, one young woman had previously been involved in drugs and gang activity. Furthermore, although the young women had some similarities (e.g., attend same school) they all had very different life stories and experiences.

Although the original criteria was that the study include three or four young women who identify themselves as Aboriginal, the other participants, the known sponsor, and myself felt that the non-Aboriginal young woman played an integral part in how this group interacts. The study could have been conducted with just the three young Aboriginal women but the key informant selected this young woman because she was part of the mentorship group. We all felt that she could provide an important and different perspective on the body-related emotional experiences that are faced by young Aboriginal women. It was clear that the non-Aboriginal woman was an important member of this intact group; therefore, all four young women took part in the study.

The young women seemed to identify with one another on many levels. When listening to the women interact, their stories made me believe that they also interacted in other social contexts. Although one participant was a quite a bit younger then the other three young women, it appeared as though they were able to easily relate. When talking about general issues surrounding the body, they young women seemed to be able to 
identify with one another. However, it was interesting to hear how some of the older participants would take more of a "big sister" approach when talking about other issues. For example, when talking about boyfriends or other life experiences, the older participants seemed to want to offer their experiences and "wisdom" to the younger participant.

When I asked the known sponsor to describe the group of young women, she described them as "very resilient". She explained how they have all experienced a lot of adversity yet they have been able to overcome this adversity and accomplish great things (e.g., three of them were very close to graduating high school). Werner (1984) has described resilient individuals as those who have confidence that things will work and that odds can be overcome. Furthermore, Benard (1993) explained how resilient individuals are autonomous; more specifically, they can act independently because they have a clear sense of who they are. The known sponsor explained that the young women of this study appeared to demonstrate these positive attributes.

\subsubsection{Phase Two: Rapport Building}

During the first meeting with the participants, I informed all of the young women of the purpose of the study. I described the proposed time frame of the study to them and informed them of the proposed methods and their involvement in phases three, four, and five. They agreed to participate and informed consent was obtained (see Appendix B). It was explained to them that each phase would take approximately 1-2 hours. Although the plan was to meet with the young women multiple times before the interview process began in order to gain trust and build rapport, it was apparent that the young women were quite comfortable speaking with one another and I seemed to gain 
their trust quickly. When speaking with the known sponsor, I told her how surprised I was that the young women were so open with me on our first day. She told me that she was not necessarily surprised because these particular young women were used to speaking with "outsiders" about sensitive topics.

The initial discussion at this first meeting was quite relaxed, consisting mostly of "everyday" conversation. The young women and I, along with the known sponsor, conversed to get to know one another better. Conversation revolved around topics such as describing our backgrounds (i.e., history). Discussing our backgrounds was a great method to get to know one another and this helped to build the context of the case. Building the context of the case involves "situating the case within its setting" (Creswell, 1998, p. 61). It was important to find out as much about the young women as possible prior to data collection so that the complexity of their body-related emotional experiences could be better understood.

Aside from building the context of the case during this initial meeting time, I also used this time to ask the young women for feedback on what they felt could be relevant methods of inquiry for the study. Stake (1995) described how qualitative studies are emergent and responsive; therefore, it was important to listen to the young women so that the issues discussed and the design of the study were responsive to the participants. Allowing for a participatory role in the design of the methods can also be empowering to those individuals "who are at the bottom of the social hierarchy" (Esterberg, 2002, p. 142). Research has shown that Aboriginal women probably fit into the "bottom of the social hierarchy", since they are "doubly disadvantaged" because they are both members of a minority group and are female (Gerber, 1990). 
In addition to empowering the young women, many feminist methodologists believe that asking for the involvement of participants encourages a less hierarchical approach by the researcher. Reinharz (1992) suggested allowing the participants to share in the decision-making, while Denzin and Lincoln (2000) recommended the involvement of participants in the development of methods so that the distance between the researcher and the researched can be lessened. By a feminist researcher abandoning an egalitarian role, shared decision-making can develop (Reinharz, 1992). Through these attempts at alleviating boundaries between the participants and myself, I cleared what I thought to be a pathway for open discussion surrounding possible methods to explore the body-related emotional experiences of young Aboriginal women.

Alleviating boundaries, and involving the young women in the development of relevant methods of inquiry, has also been supported by Olesen (2000) who added that the best way to seek knowledge about women is to start by speaking with women. Therefore, by speaking with the participants it was possible to design a set of methods which everyone deemed appropriate. I approached the young women with a very open mind of the possible methodolical direction that could be pursued. I asked the young women if they had any suggestions on the possible methods that could be used to provide insight into the body-related emotional experiences of young Aboriginal women. Since the young women did not provide me with any suggestions, I proposed a possible set of methods (i.e., focus group, one-on-one interviews, art project) that we could follow. Although I was open to possible modifications, the young women agreed that the proposed methods would be an appropriate process. Therefore, after a very informative discussion, the participants and I agreed upon the process (i.e., phases three, 
four, five) that would be used to provide insight into the body-related emotional experiences of young Aboriginal women.

The first rapport building session was successful in that the participants and I were able to develop the subsequent phases; I also seemed to have gained the trust of the participants. This was evident by our discussions and by being invited to an art show that they organized. Throughout the research process, I was also invited to different events (i.e., graduation, talent show) that the young women were involved in at their school. Gaining trust was essential to my success as the interviewer, and rapport was needed so that I could better understand the phenomenon from the participant's perspective (Denzin \& Lincoln, 1994). Furthermore, as Patton (2002) indicated, rapport was needed so that I could convey understanding and empathy without judgment, which is important since the participants expressed personal experiences surrounding feelings about their bodies. Although I felt that the young women trusted me after the first meeting, Lincoln and Guba (1985) noted that building trust is a developmental process that one needs to be engaged in daily so that the participants can be certain that confidentiality will be honored. Therefore, maintaining this trust was my first and foremost responsibility throughout this research process.

\subsubsection{Phase Three: Focus Group}

The focus group was one of the main methods of data collection. Denzin and Lincoln (2000) suggested using focus groups in feminist research because through the voices of multiple individuals focus groups are able to "reduce the distance between the researcher and the researched" (p. 641). This method of data collection may also help to empower young women by enabling them to speak with others who have had similar 
experiences (Esterberg, 2002). Furthermore, women have used conversations for decades to deal with their oppression and often appreciate the chance to express themselves during focus groups (Madriz, 2000). The purpose of this phase was not to give voice to the participants, but instead to listen to the multiple voices of the participants as the agent and constructor of the knowledge (Fine, 1994).

Although there are many challenges (i.e., confidentiality) associated with focus groups, they "have great potential for uncovering the complexity of layers that shape women's collective, and individual, life experiences" (Patton, 2002, p. 847). The stories and knowledge that are shared through group interviews have the potential to help women develop a sense of identity. Furthermore, the properties inherent in the focus group allow this tool to be a culturally sensitive method of data collection (Patton, 2002). Madriz (2000) described how women, and more specifically women of colour, can use focus groups to unveil aspects of their daily lives that are rarely researched (e.g., feelings, dreams, and hopes). The focus group was used because it can foster a sense of safety, and the young women were able to share stories that they may not have been able to do if they were in a one-on-one interview (Patton, 2002).

Focus groups usually consist of less than 10 individuals of similar ethnicity, age, and gender (Esterberg, 2002). Although not all of the young women identified as having the same ethnicity, they were all part of an intact group who felt comfortable sharing stories with one another. In order to protect the confidentiality of the participants, they were asked to choose a pseudonym to be used throughout the study. Nevertheless, the participants chose not to use pseudonyms and expressed how they were not concerned about issues surrounding confidentiality. It is possible that the young women chose not to use pseudonyms because they are proud of their identity and they did not want to hide 
this identity by using pseudonyms. Despite the reluctance of the participants to choose pseudonyms, as a researcher I recognize the importance of confidentiality and I expressed to the participants how I would respect their confidentiality throughout the whole research process.

To begin the focus group, I brought lunch so that the participants and I could relax, eat, and engage in everyday conversation. After the participants had finished eating, they spoke collectively about the female "ideals" that are often portrayed in our culture. The young women were given the opportunity to react to images from the media (i.e., popular magazines). Although I initially selected the magazines, I asked the known sponsor whether or not she felt my choices were appropriate. Also, prior to having the participants view the magazines, I asked the participants if they were familiar with the magazines and if they were their magazines of choice. All of the participants agreed. After viewing the images in the magazines, general ideas surrounding the depiction of women in the media were addressed (i.e., What kinds of images are depicted in these magazines/media? How attainable are these images to young women?; see Appendix C). The participants responded to questions from an interview guide; however, the interview guide was semi-structured and, therefore, the participants were able to openly discuss issues surrounding the body while taking the focus off of their own bodies.

The participants were then asked to create a piece of artwork that represented how they felt about their own body. They were asked to paint, write, draw, or cut out pictures from magazines of whatever they chose pertaining to their own images of how they felt about their body. Creating artwork as a method of data collection is an unobtrusive and creative way to visually capture attention (Creswell, 2003). This phase 
of the study was a chance for the participants to develop their own artwork in an attempt to express the individual emotional experiences they have with their bodies. Since issues surrounding the body may be difficult for some individuals to discuss, the focus could be placed on the artwork and not necessarily on the participant. An important goal of this phase was to build the context of the study by providing the participants with an alternative way of expressing their experiences surrounding their body. The artwork was also used as a catalyst for discussion within the focus group and the subsequent oneon-one interviews.

A semi-structured interview guide was used in this second part of the focus group and was loosely followed throughout the group interview (see Appendix D). The research questions were quite broad (e.g., Describe what your artwork means to you?) to begin with and then they became more focused on the body-related emotions as the interview progressed; this is consistent with a feminist approach. Often, feminist researchers will begin a study with general questions and these questions can be developed as the study proceeds. The intent of this study was to provide new knowledge in the area of body-related emotional experiences of young Aboriginal women and it was important to keep the questions somewhat general so that the complexity surrounding the issues could unfold. I did not want to narrow the questions too much in fear that I would not provide the participants with enough flexibility to share the experiences that they deemed important. The focus group was used as an opportunity for the participants to build on one another's ideas (Esterberg, 2002). Therefore, in this phase and subsequent phases they were free to discuss issues that were not explicitly laid out in the interview guide. 
The discussion in this phase, and throughout phases four and five, were audiorecorded and transcribed verbatim. Field notes were also taken after the focus group interview so that ideas and reflections could be recorded (Bogdan \& Biklen, 1998). After each phase, and prior to the beginning of each subsequent phase, the participants were asked to member-check the transcripts (i.e., phase three transcripts would be memberchecked before the start of phase four). In other words, the participants were provided the opportunity to add, alter, and delete information from the transcript so that the transcripts accurately reflected what the young women had intended to say during the interview. The analysis in this research project was ongoing (see section 2.1.3 for more detail).

\subsubsection{Phase Four: One-on-one Interviews}

In phase four, one-on-one semi-structured interviews were conducted, audio recorded, and transcribed verbatim by the researcher. Individual interviews were used because they allow researchers to enter into the participant's perspective, a perspective that is assumed to be meaningful, knowledgeable, and able to be made explicit (Patton, 2002). Stake (1995) said that interviews are important for case studies because the topic being investigated will not be seen the same by everyone. He also stated that interviews are one of the best ways to get at multiple realities. The purpose of semi-structured interviews is to explore a topic openly and to allow the participants to express their ideas in their own words (Esterberg, 2002).

Throughout the one-on-one interviews, certain questions were addressed in an attempt to identify the body-related emotions experienced by these young women (i.e., How would you describe your body? How do you feel when you think other people are 
looking at your body? When are you most/least comfortable with your body? What do you do when you know others are judging your body?; see Appendix E). Although the interview guide provided some guidance to the interviews, the responses of the participants shaped the direction of the interviews (Esterberg, 2002). Furthermore, since this study was approached using a feminist perspective, while utilizing CognitiveMotivational-Relational Theory as the conceptual framework, it was important to develop questions that were consistent with both. As a result the questions that were developed for this phase and subsequent phases focused on race, class, and gender, as well as appraisal and coping. Feminist researchers see gender, class, and race as principles that shape individual's lives, and this lens helped me to focus the questions on these factors (Creswell, 1998). Cognitive-Motivational-Relational Theory recognizes appraisal and coping as two key aspects of emotion (Lazarus, 1991) and therefore it was important to ask questions that address the appraisal and coping strategies.

Field notes played an integral role in the one-on-one interviews. Since the interview guide was semi-structured in nature, field notes were used to record questions that arose throughout the interviews. Also, the field notes allowed me to take notes regarding the physical setting, which could not be captured on the audio-recorder. Also, as explained by Patton (2000), the descriptive nature of my field notes helped encourage early insights and allowed me to start the data analysis the day the research began.

The time, location, and length of each interview was established so that it was not obtrusive to the participant. Each interview took approximately one hour and was conducted at a location of the participants' choice. All of the participants chose a local coffee shop, where we were served lunch, as the meeting spot for the one-on-one interviews. 


\subsubsection{Phase Five: Art Project}

The final phase was an opportunity for the participants to produce photographs, art objects, or cultural artifacts in an attempt to directly share their reality (Creswell, 2003). The young women were asked to provide some form of visual or auditory material that represented their body-related emotional experiences. This phase was consistent with other feminist researchers who encourage women to express creativity and variety as part of the research process (Reinharz, 1992). The previous phases built upon one another to provide insight into the body-related emotions experienced by young Aboriginal women. This final phase built upon the first few phases, but it was also a chance for the participants to bring in their own visual materials in an attempt to express their experiences in an individual and unique way. By giving the participants a chance to express themselves through visual (i.e., cultural artifact, photographs), or auditory (i.e., music) materials, I was provided the opportunity to try to understand the participants' experiences in a different way.

The participants and I met after phase four to discuss the procedures for phase five. I discussed with the participants the main themes that emerged from the first four phases. After member-checking the themes with the young women, they decided that they would each create a piece of art that represented one of the themes. The young women decided that they would all create a piece of art that represented a different theme. Therefore, for phase five, they were asked to bring in some form of visual material or prepare some method of expression that represented one of the themes for the body-related emotional experiences. It was explained to the participants that they 
would not be judged on the quality of the form of expression. The goal of this phase was to provide some form of expression that would generate discussion.

For example, the participants could have brought in cultural artifacts or pictures that adequately represented a particular theme. Cultural artifacts may have been used to share their reality (Creswell, 2003) or the photographs could have been used as a visual representation of the participant's everyday world (Denzin \& Lincoln, 2000). Denzin and Lincoln (2000) recognized that photographs could be used to understand the emotional intent of the photographer. Although two of the participants did not create an art project due to other commitments, two of the participants chose to do picture collages (see Appendix F). One young woman used personal photographs of herself, family, and friends. The other young woman used many different pictures from fashion magazines and she also created a poem to go along with the collage. In an attempt to generate discussion, they were asked questions about the item that they chose to bring (How did you chose this piece of work? How does this piece of work represent how you feel about

your body?; see Appendix G). Through the use of field notes, I was able to record specific descriptions of the young women's artwork that they described. Although the discussion was audio-recorded, the young women often pointed out specific aspects of their art and this was important to record through field notes.

\subsubsection{Data Analysis}

In my analysis, I do not seek to describe the world or even to describe fully the case. I seek to make sense of certain observations of the case by watching as closely as I can and by thinking about it as deeply as I can (Stake, 1995, p. 77). 
The analysis of the data began the day the research process began because analysis involves not only the final compilations but first impressions as well (Stake, 1995). Qualitative data analysis cannot be described as one distinct process, and Patton (2002) has indicated that there is no data analysis formula that exists with regards to how to transform qualitative data into findings. Stake (1995) described how case study analysis is not standard and each researcher must find a way that best suits him/her. Although a range of guidelines for data analysis of case studies does exist (Creswell, 1998; Stake, 1995; Yin, 1994), it is important that researchers draw upon their own creativity and judgment (Patton, 2002); thus, data analysis is unique for every researcher (Stake, 1995).

Having many similarities to thematic analysis, I used Stake's (1995) guidelines for analyzing this case study. Stake described how direct interpretation and categorical aggregation should be employed to search for new meanings. The transcripts and field notes were analyzed; the field notes helped to situate certain quotes within the transcripts so that I was better able to understand the context within they were spoken. Creswell (1998) suggested using direct interpretation to examine single instances for meaning, without looking for multiple instances. As stated by Creswell, I was basically attempting to pull apart all the data from each of the phases and trying to put the information back together in a meaningful manner. Categorical aggregation, on the other hand, was utilized to find issue-relevant meanings by searching for a collection of instances from the data (Creswell, 1998). Direct interpretation and categorical aggregation were utilized in the first part of the analysis, and are relied heavily upon by case study researchers (Stake, 1995). 
The next step in the analysis of the data was what Stake (1995) refers to as correspondence and patterns. Creswell (1998) described this stage as a chance to create patterns, and subsequently, look for a correspondence between the patterns. Stake stated that the search for patterns is derived by the search for meanings; patterns are those descriptive findings that are located throughout the data. Patterns were then grouped together to form themes (Patton, 2002). It is important to note that I recognize that researchers bring their own individual perspective when interpreting data and even though I member-checked the themes with the participants, the themes are still my own interpretation of participant experiences.

Lastly, Stake (1995) indicated that one of the final steps of case study research is to develop naturalistic generalizations. People can use these generalizations so that they can learn for themselves or use the information to apply to future cases (Creswell, 1998). Naturalistic generalizations differ from traditional scientific generalizations in that they are conclusions that are derived from vicarious experience (Stake, 1995). Case researchers must provide the chance for the expression of vicarious experiences so that cases can become understandable and generalizations can be made (Stake, 1995). Stake described how narrative accounts foster the opportunity to express vicarious experience and, therefore, the words of the participants are used throughout the results section.

Feminist theory and Cognitive-Motivational-Relational Theory were utilized throughout the data analysis process when I was trying to interpret, and bring meaning, to the findings. I sought all possible meanings with regards to the case and then constructed a thesis that I felt best captured the meaning and the essence of the young women's body-related experiences (Creswell, 1998). Themes are presented in the results section (see section 3.1) with supporting quotes from the participants. The words 
of the participants were used because the stories that are told offer a clear look into particular social and cultural meanings (Patton, 2002). "Story telling, oral histories, the perspective of elders and of women have become an integral part of all indigenous research" (Smith, 1999, p. 144); therefore, it was important to try to tell their stories through the use of long and short quotes.

Many indigenous writers share stories as a means to convey the beliefs and values of a culture (Smith, 1999), and it is for this reason that the words of the participants were used within this project. It was important to include the experiences of all of the participants since each individual story is powerful (Smith, 1999). Feminist researchers assume that the words of participants cannot be taken out of the cultural context from which they are rooted (Way, 2001). For example, when the participants used certain words (i.e., Native as opposed to Aboriginal), I was sure to represent their words as accurately as possible.

\subsubsection{Verification}

Many procedures were used throughout the research process to verify the findings of the study. Olesen (2000) acknowledged that feminist qualitative researchers could use a variety of methods (e.g., rich thick description) to verify the findings of a study, depending on the way they frame their research approach. Creswell (1998) recommended utilizing at least two methods of verification. Stake (1995) indicated that case studies require extensive verification and identified triangulation and memberchecking as two possible methods. However, since Creswell has indicated that many of the procedures (i.e., member-checking, triangulation) are cost-effective and easy to conduct, it is often possible to use more than two methods of verification. The methods 
of verification that were undertaken within this study included prolonged time in the field, triangulation of methods and investigators, member-checking, the use of rich-thick description throughout my thesis, and the employment of Stake's critique checklist.

\subsubsection{Prolonged Time}

This study was organized into five phases, which allowed for prolonged time in the field. I met with the participants four times in the more formal phases of the study and then I met with the participants in other less formal situations (i.e., art show) approximately four other times. I spent prolonged time in the field to try to learn the context within which the participants live (Creswell, 1998). In addition, prolonged engagement was imperative to build adequate trust and rapport between the participants and myself (Lincoln \& Guba, 1985). By spending prolonged time in the field, I was able to express detail about the participants within my thesis, which added to the credibility of the findings (Creswell, 2003).

Time was spent getting to know the known sponsor, as well as the participants, in multiple informal meetings. Furthermore, the study consisted of multiple data collection phases. By spending a period of extended engagement with the participants, I tried to demonstrate that their confidences would never be used against them (Lincoln \& Guba, 1985). Although the literature (e.g., Creswell, 1998; Lincoln \& Guba, 1985) states that it is important to spend a prolonged period of time with the participants, it was also important to respect the fact that the participants were volunteering their time to the project. 


\subsubsection{Triangulation}

Triangulation of methods and investigators are often used in case study research to support the credibility of the findings (Stake, 1995). Methodological triangulation took place by utilizing multiple methods to study a single phenomenon (Patton, 2002). Focus groups, one-on-one interviews, art elicitation techniques, observation, and field notes were used to try and understand individual emotional experiences with regards to the body. Since each method has strengths and weaknesses, I felt that it was important to include various methods. Lincoln and Guba (1985) summarized the benefits of using triangulation of methods by stating, "It is as though a fisherman were to use multiple nets, each of which had a complement of holes, but placed together so that the holes in one net were covered by intact portions of other nets" (p. 306).

Investigator triangulation was also used during the data analysis. Esterberg (2002) indicated that if multiple investigators arrive at similar conclusions with regards to the data, the primary investigator might feel more confident with the accuracy of the findings. The process of triangulation allowed for multiple investigators to shed light on a particular phenomenon (Creswell, 1998). Although my research advisor and graduate committee members each played a role in the triangulation of investigators, they each had distinct roles. For example, my research advisor would continually ask questions throughout the research process which pertained to methods and interpretations (Creswell, 2003). He also read through all of the transcripts and engaged in the ongoing data analysis. He would ask questions so that the meanings that were developed could be explored, and so that the biases and interpretations that arose could be explained (Lincoln \& Guba, 1985). In addition to my research advisor, my graduate committee members also played a less involved role. Basically, my committee members would ask 
more general questions involving the methodological, ethical, or any other issues pertaining to the study that may not have been clear (Lincoln \& Guba, 1985).

\subsubsection{Member-checking}

Member-checking was used as another method of checking the accuracy of the findings. Some researchers (e.g., Lincoln \& Guba, 1985) view this technique as the most essential method of establishing credibility. All transcripts and proposed themes were given to the participants for feedback as to whether they felt the findings were accurate (Creswell, 2003). Participants were encouraged to change, delete, or add information to the transcripts as seen fit. It was essential that all participants be given the opportunity to react to the transcripts (Lincoln \& Guba, 1985). Prior to writing my thesis, it was important for me to give the participants the opportunity to react to the transcripts so that I could confidently say that the findings represented the participants' own "realities".

\subsubsection{Rich, Thick Descriptions}

The findings were conveyed throughout my thesis with what Lincoln and Guba (1985) referred to as rich, thick descriptions. The context of the case study was developed and explicitly described in my thesis. This sort of information-rich description allows readers to decide upon the degree of transferability of the findings (Creswell, 1998). Furthermore, conveying the information in such a descriptive manner fostered a thesis in which the readers are hopefully able to gain a sense of shared experiences (Creswell, 2003). Although external validity of any inquiry is not 
something that can be specified by a naturalist, thick description can allow readers to come to their own conclusion with regards to the data (Creswell, 1998).

\subsubsection{Critique Checklist}

Lastly, Stake (1995) provided a "critique checklist for a case study report"(p. 131; see Appendix H). This checklist was employed as the final method of verification and it includes 20 criteria for assessing a case study report. Questions that include, "Is the report easy to read?; Is the case adequately defined?" (p. 131) were asked, and then I was able to assess the overall quality of the case study. The checklist was completed at the end of the study. 


\section{CHAPTER 3}

\section{1 RESULTS}

The purpose of this study was to provide insight into the body-related emotional experiences of young Aboriginal women. The stories of the young women are presented collectively because it was a collective case study and the strength of the study lies in the overall story of these four young women. In general, the young women noted a sense of satisfaction or happiness with their bodies, which will be discussed within the various themes. The five themes that emerged and will be discussed throughout the remainder of this thesis are conflicting cultures, need to belong, personal identity, journey to acceptance, and the body affects everything. The words of the participants and the themes have been member-checked.

\subsubsection{Conflicting Cultures}

One of the major themes that arose was the idea that young Aboriginal women are often faced with conflicting cultures. The two cultures that the participants viewed as being in conflict were their own Aboriginal culture and the urban white culture within which they live and go to school. They described how the beliefs that are held by their Aboriginal culture are often quite different than the beliefs of those people in mainstream white culture. They explained the difficulties faced by young Aboriginal 
women who cannot look like or fit into the majority white society. One participant explained:

Their body, the way that they feel about their body. The fact that they can't afford these clothes, the fact that they'll never look like a white person, the fact that their skin will never be that light or their waists will never be that small...brings on a lot of jealousy and causes violence ... you know how there's that culture clash all the time.

Some of the participants spoke about the difficulties they face when they go back and forth between their urban residence and their home reserve. Their home reserve often has different views than those views held by people in the city with regards to issues surrounding clothing, body size, and eating habits. One participant spoke of the difference between what was considered to be appropriate clothes to wear when on her reserve and when in the city. She said that a certain outfit may be deemed as "skimpy" by the elders but "in the city I could be wearing this little t-shirt and a pair of shorts and it's considered normal". She further explained that when on her reserve she would not be able to wear a tight $\mathrm{t}$-shirt or a particular hooded sweatshirt because it had a small embroidery of a Playboy bunny sewn onto it. Nevertheless, this young woman was wearing the outfit during our interview and had just come from her school in the city; thus indicating that it was appropriate wear for the city.

When I asked the young women to explain why they were not supposed to wear certain outfits on their reserve, they said it was because some people may classify them as sex objects by the clothes they wear. One participant added, "In my culture, it is so disrespectful to your family and to yourself to even let yourself be portrayed like that (a sex object)". The young woman continued on by saying that it does not matter whether 
the young women are on their reserve or in the city, they should not portray themselves like that at anytime. She explained how "you don't even have to have Elders around you, you can be doing anything anywhere, and everybody knows about it". She said that she can be dressed a certain way, or doing something, in the city and everybody on the reserve knows about it. Although it initially came across that this irritated her, it became quite apparent that she was proud of the connectedness that she shares with the people on her reserve. It seemed apparent to me that the support that she received from her Aboriginal community played an integral role in her life.

Differing views on what is deemed appropriate wear was only one aspect of the conflicting communities. The young women also spoke about the conflicting beliefs about body size and eating habits. One young woman explained:

I find that a lot of people are really white around here, they're all concerned about their weight and everything; but out in the reserve you can stuff your face and nobody would care.

When talking about her reserve another participant added, "everybody is like overweight. And if you're not overweight they're like 'you're so skinny, you're so skinny'. And they bug you."

In addition to the conflict surrounding the amount of food that was "appropriate" to eat, some of the young women described the conflict surrounding the types of food they ate. One participant explained that when she was younger she would conceal the fact that she ate rabbit stew because this was not the norm in mainstream white society. The participant would eat the rabbit stew when she was with members from her Aboriginal culture but when she was surrounded by those from outside this culture she would adjust to the behaviors that were common to all members of the white society. When talking 
about eating rabbit stew to those people outside her Aboriginal culture she would say, "my family does. But I don't. I think that's gross".

The young women continued to explain how the views of people on their reserve are often quite different than the views of others in mainstream white society. One participant explained how she felt when she was on the reserve, "I feel like it's totally opposite than everybody else." The young women were then asked how they felt when they were faced with conflicting views between their Aboriginal culture and urban white culture about what was considered right or wrong with regards to appropriate wear, body size, or eating habits. One of the participants responded, "It just makes you hate your body even more...it's kind of this constant battle with yourself'. The young women continued to speak about how they were forced to make their own decisions regarding the above-mentioned issues during adolescence, a time they already labeled as being a tough time. The young women spoke of the difficulty of this decision making, especially for young women, because it's "at the critical point in your life where you already have all this puberty and all these different things hitting you".

Although the young women spoke a lot about the conflicting cultures when talking about their Aboriginal culture and urban white culture, some of the young women recognized that there were differences across the many reserves as well. For example, they said that the reserves that have a lot of money care more about physical appearance and everything physical, including the vehicle that they drive. One young woman said that maybe it is money that makes cultures conflict with one another. Similarly, the known sponsor said that maybe young women experience things differently because of socio-economic status and not necessarily their race. Money was seen as an issue across reserves (i.e., "rich" reserves versus "poor" reserves), but it was 
also an issue between their Aboriginal culture and the urban white culture as well. The participants explained the importance of money and the role it plays in appearance management. For example, one participant explained how she felt when she' was younger and went to school with children who had more money than her, "I lived on the east side and I always saw all these designer clothes and designer bodies... and I couldn't afford all these clothes".

The one participant who did not identify herself as Aboriginal had an interesting perspective surrounding this notion of conflicting culture. She explained how she has a friend who is black and "they're (black people) kind of like that too...'oh you need to gain some weight' and...I don't think I've really seen any white families that are like that". She explained how the pressures for thinness and the focus on the body might just be something that white people are more "susceptible" too. Interestingly, the other young women in this study also supported this belief.

Conflicting cultures was a theme that the young women spent a lot of time speaking about. The young women spoke about the conflicting views surrounding appropriate wear and body size held by people in their Aboriginal culture and their urban white culture. The young women also explained how the various reserves are often in conflict with one another. Most of the young women felt that the conflicts that were experienced were the result of one culture having more money than the other. Nevertheless, all of the young women noted some degree of conflict between cultures surrounding body issues. 


\subsubsection{Need to Belong}

The need to belong was a theme that became increasingly clear throughout the interviews; all of the young women articulated this point in some way. The young women all noted the importance of belonging and wanting to fit comfortably within a community (i.e., social identity). One participant described, "the belonging...how we can never be like white people". The view expressed by some of the young women that they could never be like "white people" indicated that they may never feel totally comfortable within white society, or that they may never be able to socially identify with white people. Another young woman explained how she formed her social identity: "we moved to my side, to more native people... then finally I got to be around my own people... I was more comfortable and more happy". She explained how she felt like she belonged when she went to live with her "own people" and the constant worry of not fitting in with white people became less of an issue. Although these concerns were not fully eliminated, she no longer had to face these concerns on a daily basis.

In addition to the idea that some young Aboriginal women do not feel like they can ever belong to white society, one of the participants explained how young Aboriginal women might experience this need to belong more than young women of other cultures. She said this need to belong might be even stronger with young Aboriginal women because Aboriginal youth often do not have the mother, or father, figure that many other youth have, resulting in a desire to belong to "something". She explained that when they belong to something (i.e., a gang) they then belong to their "own little family type thing. They make you feel better than you would if you were just alone". She described how important it is to belong to something so that you are not worrying about your weight or "trying to be like everybody else". She explained: 
Cause when you are with somebody else, you feel strong enough to say I don't care. I don't care what I look like. I don't care how much money you have. I don't care what I eat. I like eating this, and so do the rest of my friends.

In her words, belonging means that you have support. You are not alone when you are facing issues regarding what you look like or what you eat. Having similar beliefs to friends or family helps to take the pressure off of oneself.

When speaking about the importance of belonging, one young woman explained that how a person looks is a major component of belonging. This particular participant identified herself as Cree; however, she said she was one of the only people in her family who had blond hair and blue eyes. When speaking about the need to belong she explained, "sometimes I wish I had the dark hair, the dark skin...then other times it's like, okay, I fit into what society calls normal". She said that she wished that she had the dark hair and dark skin because then she would feel like she belongs to her Aboriginal community. Interestingly, the young women's notion of what is considered "normal" is consistent with mainstream white society's depiction of the ideal beauty: white skin, blond hair, and blue eyes. The young woman explained how she sometimes does not feel like she belongs to either community. Physically she does not look like the rest of her family who are Cree; on the other hand, she is Cree and does not necessarily fit into white society because of her Aboriginal cultural beliefs. Nevertheless, she recognized that it may be easier to look "normal" or look like a "white person" when living in a non-Aboriginal urban community. In addition, the one young woman who did not identify herself as Aboriginal stated, "pretty girls go with pretty girls, or the groupie girls go with the groupie girls". In other words, people are grouped together based on 
similarities, and it can be difficult for those individuals who do not feel like they are similar enough to belong to any group.

\subsubsection{Personal Identity}

Personal identity was another theme that emerged from the interviews. Although the participants did recognize the importance of belonging (i.e., social identity), they also noted the importance of personal identity; the importance of being different and having a sense of separateness (Hewitt, 2000). Without using the terms personal or social identity, one young woman summed up her thoughts by stating the following:

Lots of people don't ever get the chance to develop into their own personality really. You know, like some people are social their whole lives and going with the grain their whole life; they never actually develop what they really believe.

This theme of personal identity became evident to me when listening to how the young women felt about their bodies; "well I am 100\% satisfied with my body, even if I was bigger or smaller, because I know that I'm different". Furthermore, although fearing to sound somewhat cliché, one participant explained how "not everyone's perfect, everyone comes in different shapes and sizes, and everyone's different".

Although all of the young women recognized the importance of being different, they did not try to negate the idea that appearance, and how one looks, are still important. Nevertheless, they realized that beauty is more than just what the media usually depicts. One young woman said that it is important for young women to take a close look at themselves and try to find the beautiful traits within themselves. When I asked how one young women went about doing this she added, "I stopped thinking about trying to be like everybody else and I just started to conform into my own body, 
you know, started fitting my own body". The young woman who did not identify herself as Aboriginal had similar views. When I asked her what she would like other people to see when they look at her, she said her own "individuality" as opposed to physical traits.

Adding to this notion of individuality, another participant described how she felt through the use of art. In her final art project she created a collage using pictures from various fashion magazines (see Appendix F). Trying to represent the importance of individuality and difference, she chose pictures of women who all had different physical appearances (i.e., different skin colours, body shapes, hair colours). This young woman also expressed how she felt by creating a poem in which she said beauty should come from within as opposed to the exterior appearance as so many people believe. She said that all women are different and, as a result, it would be too difficult to say what physical features equate beauty.

Personal identity, or as some of the participants called it, individuality, was something that all of the young women viewed as important. One young women explained how she used to be embarrassed to tell people that she is Aboriginal. She said that when she was younger, she thought that white society did not have "really good thoughts or feelings about them (referring to Aboriginal people)". She further explained, "but then I just learned to accept it because that's who I am. And no one, nobody can take that from me...I was made this way for a reason". It is possible that the young women in this study chose not to use pseudonyms because they accept, and are proud, of who they are and they did not want to hide their identity by using pseudonyms.

In addition to what the young women had to say about their personal identity, one young woman created a piece of art, during the final art project, that represented her personal identity (see Appendix F). She created a collage using personal photographs. 
Interestingly, she said that her personal identity is made up of more then just how she looks. She said that her family (i.e., aunties, cousins, boyfriend) is a major component of her personal identity. Although this young woman did not describe family as a major component during the focus group or one-on-one interviews, family emerged as an important aspect of personal identity during her final art project.

Lastly, and consistent with the "Indigenist" Stress-Coping model, I thought it was important to discuss the unique strengths that they felt they possess as young Aboriginal women. One young woman stated, "we are all in tune with our spirits". When I asked her to further clarify what she meant, she explained how people in her culture would go to round dances and Powwows and that these events would be very spiritual. Not necessarily contradicting the views of the other young women, one participant pointed out that "every culture is unique". Therefore, although someone may identify themself as Aboriginal, it does not necessarily mean that all Aboriginal women share the same views. It is clear from the stories of the young women that we cannot assume that there is just one Aboriginal identity. Although they may identify themselves as Aboriginal, the values, beliefs, and even physical traits of the each young woman were different.

\subsubsection{Journey to Acceptance (of the body)}

Another theme that emerged during the data analysis was the notion of a journey to acceptance of the body. The young women recognized the importance of acceptance and how a journey to acceptance takes place for many. All of the young women indicated that they currently had a general level of satisfaction or happiness with their bodies. When I asked the participants what happiness meant, one participant said that 
"happy is a 10 out of 10". Another participant explained, "I got 10 fingers, I got 10 toes, two legs, two arms, a head, and a couple of ears. I'm good to go." However, although the young women appeared happy with their bodies, they all recognized that their bodies were not "ideal". One of the young women said that although she was a "little bit overweight... that's okay". She continued on to say that she was satisfied with her body but "my boobs look really big and that sucks sometimes". Another participant said "there is nothing bad and there is nothing really great. It's just kind of average". Even after one participant described herself as completely happy with her body, she recognized that everyone has flaws, and that she was not perfect.

The young women said that although they recognize that they may not be perfect, this does not mean that they are dissatisfied with their bodies. When asked to respond to statistics that indicate that over $50 \%$ of young Aboriginal women are dissatisfied with their body (e.g., Story et al., 1995), one participant explained that she did not believe that young women were dissatisfied with their bodies. Instead, she said they were dissatisfied with the clothes that they have to wear. She explained, that it is not that people are dissatisfied with their body it is just that they "have to get these clothes that make your body look good, that accent certain features...it's mandatory. Because if we were all nudists it wouldn't matter...our faces are allowed to be nude". She further explained that this is why many young people are happy with their faces and not necessarily their body; their faces do not have to wear anything.

Although all of the young women said that they were happy with their bodies, they recognized that they have not always felt this way about their bodies. When I asked how they came to feel this way (i.e., happy) about their body they said, "just accepting it...having to look at yourself everyday in the mirror, and you either fall into this deep 
depression of hating yourself or you just say screw everybody. Why am I going to let them make me hate myself?" Similarly, another young women explained, "who am I to say that I'm not pretty enough or I'm not skinny enough...like look around me, how selfish is that for me to sit here and say that I'm not pretty enough".

When I asked them if them if they have had any influences (i.e., media, friends, family) in their lives that have helped them come to accept their body most of the young women said that they did it by themselves, "just going over it a million times in your head...until you really believe it". However, the participants did note that if they had to pick any influence it would mostly be people in their family and their friends. One participant even noted the importance of books in this journey to acceptance. She said that she read books such as 365 Ways to Be Happy so that she can surround herself with positive thoughts.

The participants were able to provide a lot of insight into this journey to acceptance. As one participant described, "when I was 16 it would have been a constant battle...with my emotions and my body and ...trying to feel good about your body...it was just a constant battle of finding clothes that fit you nicely. But my body, I was happy with it". Another participant added, "I was like forget it, I'll wear whatever makes me look good...and that's what I did, created my own style". Although the young women recognized that style and trying to make their bodies look nice in certain clothes was often a battle, they still ultimately have come to a point of acceptance about their bodies. Acceptance was not something that was restricted to the older participants; even the one 14-year-old young woman described how she has learned to accept her body. She explained, "I used to get offended when people called me crystal cracker (referring to a white person)" because she said "I'm not a cracker (referring to her light 
complexion)". Nevertheless she said she just stopped caring. Similar to some of the discussion by the other young women, she said she learned to accept her own body by "not caring" what others thought.

\subsubsection{The Body Affects Everything}

The initial idea for this final theme was derived from one young woman who proclaimed, "the body affects so much more then people think; like it's unbelievable. If we could all as a society get past the body-thing". After hearing her statement, I began to look more closely at this notion that the body affects everything. I was somewhat surprised at how much the young women said that their bodies, and their emotions, influence them. Prior to describing the many ways in which the young women felt their bodies affect them, it is important to note that the emotions that the young women experienced were very context specific.

The young women in this study provided many examples of the ways in which their emotions would differ depending on the specific situation. For example, one young woman said that she felt good about her body when she was Irish or Powwow dancing because people are watching her and she knew that "they're supposed to be looking". However, if she was walking down the street and people in the cars were looking at her body then she said she might feel uncomfortable. When another participant was asked how she would feel if people were looking at her body, she said that it would depend on the "kind" of judging that was taking place. She said that we do not always judge

people in a bad way. We may be looking at, or judging, a person because we think they look nice or are envious of certain traits that they possess (i.e., nice body). 
The participants also noted that they might feel uncomfortable if it was "old people" looking at their body as opposed to young men their own age. One participant explained, "you know that it's not right when a 60 -year-old is checking you out or watching you bend over". Aside from the different situations that the participants are often faced with, it was also noted that young women might have concerns about their bodies if they are in a "bad mood". They said that if they were in a really good mood and they were having a bad hair day then it would not matter. However, if they were in a bad mood, they would probably care about their hair. Despite the inherent limitations in trying to understand someone's emotional experiences and the context specificity of their experiences, the young women did describe how their body-related emotions affected many aspects of their lives. For example, one young woman said that, "if you are not happy with yourself then you're not going to be happy with anything".

Although I was not initially sure what she actually meant when she said that young women would not be happy with "anything", the young women helped to clarify it for me. Not only does the body and the related emotions affect how one feels overall, but it can also influence one's behavior and the choices that are made. One young woman described an experience from when she was younger; "I got into drugs, and that helped me lose weight at first. And I was like cool, maybe I should just keep doing this you know to get more skinnier and more skinnier". She thought that doing drugs would help her to be skinny, therefore, happier with herself. Although she finally recognized that drugs would ultimately have to be traded off for her overall health, the idea of staying on the drugs was definitely something that was considered.

Other behaviours that the body-related emotions affect are coping behaviours. When asked how they felt when others were viewing their body, one young woman said 
she felt uncomfortable. She continued by saying that she would probably just walk away and forget about it because they're probably not "worth my time". Another participant said, "I look at them and I'll whisper and I'll pretend I'm talking about them". Another participant took more of a direct approach and said that she might ask the other people why they are looking at her.

Aside from the choices that are made about certain behaviours, the young women described how they sometimes make choices about friendships based on physical appearance. One of the participants explained, “you don't want somebody coming with you somewhere that is massively overweight, grossly overweight, and everybody else is thin and you have this nice looking group, and then there's this one overweight person... and it's so mean but it's true". Although this young woman recognized that it was "mean" and "not right" to think this way, she explained that in a social setting they may be ostracized for socializing with such people. She said that it is possible that people (i.e., boys) might not want to approach them, talk to them, or invite them to parties if they are socializing with someone that is extremely overweight.

The young women also described how jealousy is often a result of how young women feel about their body. They pointed out that how someone feels about their body would determine whether or not they are jealous. Although they recognized that jealousy could be derived from a number of things, the young women claimed that the body was a major reason for jealousy. In reference to jealous girls, one participant said, "they can't look like those other girls...because they are who they are and they can't change that... and sometimes they can't accept that". She continued by saying that if people do not accept themselves, they become jealous. Another young women indicated that the pressures to be pretty cause girls to become jealous. 
The body also has an affect on many young Aboriginal women because the body often determines who will be represented in the media. Since Aboriginal women typically do not fit the media portrayed depiction of beauty, there are relatively few, if any, Aboriginal role models that are being presented to the young women in the media. Throughout the focus group sessions and one-on-one interviews, some of the young women spoke how young women are lacking Aboriginal women role models in the media, recognizing that this is probably because they do not fit the media image "ideal". The young women spoke how Aboriginal people, and women more specifically, are playing predominant roles in society but are not being recognized. With regards to the lack of Aboriginal women representation in the media, one young woman said, "they are not even letting us have role models. They wont let it". When the young women were viewing the pictures in the fashion magazines during the focus group, I asked the young women to describe how the lack of Aboriginal representation made them feel. Words such as "small"and "tiny" were common descriptors.

Although three of the young women agreed that there was a lack of Aboriginal representation in the media, one participant held a different opinion. This participant, although she identifies herself as Aboriginal, has very few, if any, features that she would claim as Aboriginal physical traits. She is currently a model and she said "some of them (models) might not be full blown but they still do have it in them". She recognized that there may not be many women who carry certain physical traits of Aboriginal people, but they are still Aboriginal. She recognized that there is more to being Aboriginal than just looking Aboriginal; the physical aspect is just one component. 
Since the young women described many aspects of their lives that the body affects, I was interested in finding out what the young women felt should be done to deal with these experiences. One young woman explained, "there should be young peoples groups, like an organization. Have rallies towards freedom of body and then all these other young people would see it." She continued on to describe how this could be a public place for young women to go where they would not have to care about their body. Another young woman said that we need to make Aboriginal women more "prominent in society". She felt that Aboriginal women were doing important things in society and they were not being recognized. She felt that if the media recognized the accomplishments of young Aboriginal women, than other young women would be provided with role models.

\subsection{DISCUSSION}

The purpose of this study was to provide insight into the body-related emotional experiences of young Aboriginal women. A general level of satisfaction with the physical-self was experienced by all of the young women and will be discussed as one of the main findings within this study. The other main findings that will be discussed are the journey to acceptance, conflicting cultures, need to belong, personal identity, and the body affects everything. Furthermore, feminist perspective and Cognitive-MotivationalRelational Theory will be integrated throughout the discussion to guide the interpretation of the results. 


\subsubsection{Main Findings}

All of the young women in this study experienced satisfaction with the physicalself and this finding is inconsistent with most of the previous research that has examined young Aboriginal women's body-related emotional experiences. Although the young women all noted a general level of satisfaction or happiness with their body, as

previously stated, Gittelsohn et al.'s (1996) quantitative study found that only $16 \%$ of the young women from an Ojibway-Cree community in Northern Canada were satisfied with their bodies. Similarly, numerous American quantitative studies (e.g., Rinderknecht \& Smith, 2002; Story et al., 1994) have shown similar levels of body dissatisfaction among young Aboriginal women. Although the young women in this study may be part of the reported small percentage of Aboriginal women who are satisfied with their bodies, this study utilized qualitative inquiry in which the complexity of the young women's emotions could be expressed. Therefore, it is possible that the young women view their bodies as being "good enough" as described by Rand and Resnick (2000). Furthermore, it is possible that the young women in this study felt similarly to those women interviewed by Thompson et al. (2002). They found that American Indian women did not feel pressured to look slim and thus they were less competitive about physical appearance than the non-Hispanic white culture.

The feminist perspective that was taken in this study played an important role in providing the young women the opportunity to express their feelings of satisfaction with their body. This perspective assumes that the path to understanding is based on the experiences of women and listening to their stories (Way, 2001). Although previous research (e.g., Gittelsohn et al., 1996; Story et al., 1994) has indicated that young Aboriginal women are dissatisfied with their bodies, the young women in this study 
were able to share their stories and express how their experiences may have been different to those young women from previous studies. Through their words the participants were able to describe their complex body-related emotional experiences.

The findings from this study are consistent with Cognitive-MotivationalRelational Theory because this theory recognizes that emotion is a complex context driven process (Lazarus, 1991). The young women had a hard time explaining how they felt at all times because their emotions would vary depending on how they appraised situations. For example, if "old men" were viewing their bodies, they would appraise the situation as threatening and experience negative emotions (i.e., anger). However, if "hot guys" were viewing their bodies they would experience more positive emotions (i.e., happiness). Therefore, the emotions of the young women were very context specific.

Cognitive-Motivational-Relational Theory also recognizes that there are various emotions that a person can experience, and Lazarus (1991) has provided a detailed list of the many possible emotions. All of the emotions (e.g., happy) that were described by the participants were among the list that has been provided by Lazarus; however, the young women often used terms that were more broad to describe their emotions (e.g., satisfied). Nevertheless, the emotions that the young women did describe were consistent with the respective core relational themes and patterns of appraisal that were described by Lazarus. For example, the core-relational theme of happiness is that we have gained or are in the process of gaining what we desire. The young women expressed how they were happy and/or satisfied with their bodies because they like the way they look. In other words, they have achieved their desired look. 
While the young women in this study noted that they were satisfied with their bodies, there were still some parts of their body that they were not totally satisfied with. As stated in the results section, one young woman was satisfied with her body in general but she was unsatisfied with her breast size because she felt that they were too big. This is consistent with Sherwood and Neumark-Sztainer (2001) who found that although most of the young women in their study were satisfied with their bodies, many of them were dissatisfied with specific body parts (e.g., lower body). Furthermore, Monteath and McCabe (1997) have said that self-report questionnaires have shown that women are consistently dissatisfied with specific body parts, but are not uniformly dissatisfied with their body appearance.

All of the participants in this study reported being currently satisfied or happy with their bodies; however, some of the participants recognized that they might not have always felt this way. It is interesting to note that even though the young women were able to currently accept their bodies, their body had not changed much since the time when they were less happy with their bodies. Therefore, it is quite possible that the current satisfaction with the body was the result of a journey to acceptance in which young women come to accept their body for what it is. Heilman (1998) said that many theorists believe the self is fluid and changing depending on circumstances; the stories from the young women in this study support this belief. For example, the young women in this study explained how they are constantly faced with media created images of ideal beauty and it may be the media that influences whether or not they come to accept their bodies.

Being faced with particular circumstances, such as media created images of ideal beauty, might enable young women of colour to learn to reject the media portrayed 
notion of beauty and accept their physical-self for what it is (Rubin, Fitts, \& Becker, 2003). Rubin et al. (2003) have shown that young black and Latina women note the importance of self-acceptance and the rejection of mainstream societies pressures to conform to the "ideal body" that is supported by the media. Although the participants in Rubin et al.'s study were black and Latina women, the young women in this study contested the notion that beauty can only be equated to slimness and whiteness. Furthermore, Cash (2002) explained how young women who believe that the physicalself is personally acceptable will most likely come to believe that their body is socially acceptable (Cash, 2002). Having a body that is socially acceptable may then allow for more positive body-related experiences (e.g., body satisfaction). Therefore, the journey to acceptance of the physical-self experienced by the young women may be a result of both personal and social acceptance.

In addition to the journey to acceptance, the young women spoke about the conflict often experienced between their Aboriginal culture and mainstream white society. They explained how stressful it could be when there are discrepancies between the two cultures on what are appropriate body sizes, foods to eat, and clothes to wear. The idea that the young women experience conflicting views on appropriate body sizes is consistent with Gittelsohn et al. (1996) and Thompson et al. (2002) who found that Aboriginal women are more accepting than non-Aboriginal women of larger body types. Furthermore, when discussing the conflicting views in more general terms, these findings are consistent with Hewitt (2000) who said that it is often difficult to live up to the images of an enclosed ethnic community (i.e., Aboriginal community) when these images are not consistent with the dominant members of the society in which they live. As summarized by Kirmayer et al. (2000), the notion of Aboriginality is sometimes 
thought of as a "dividing practice" that marginalizes and unites. Hewitt wrote how some individuals may try to conceal peculiar ethnic habits when interacting with a nonminority society. Therefore, it is not surprising that one of the participants used to conceal the fact that she ate rabbit stew when she was interacting with non-Aboriginal people. Hewitt further explained how we often rely on the standards of the community to which we belong but this can be very difficult for those individuals who find themselves torn between two communities. One participant referred to this as the "culture clash" because the standards between both cultures are often so different.

In addition to the notion that the young women felt that their Aboriginal culture often clashed with white society, it was interesting to hear the terms that the young women used when describing the conflicting groups. When the young women spoke about their "culture", they spoke about it in a positive way that signified unity and support. However, the young women often spoke about white "society". Although it is unclear as to whether or not the young women intentionally used different terms, Hewitt (2000) said that the term society is often used to designate a larger social entity where the members of this entity feel little loyalty towards one another. Furthermore, he described how the term society is often viewed as bad. Whether or not the young women knew this when they were talking about their Aboriginal culture and white society, it is a point worth noting because there may have been some underlying connotation.

In continuation of the discussion of the conflicting cultures, the "Indigenist" Stress-Coping model can help us to better understand the experiences of the young women. As previously discussed, the young women noted how there are conflicting beliefs between their Aboriginal culture and the urban white culture within which they 
live. By incorporating the "Indigenist" Stress-Coping model, it became clear that these problems might be associated with the fact that many of these young women are living in areas in which a subordinating majority (i.e., white people) holds the power (Walters \& Simoni, 2002). Therefore, the negative experiences of the young women in this study might have been a result of their beliefs differing to those of the majority. Similarly, Wilson and Rosenburg (2002) have also noted how Aboriginal people who live in urban centers might experience additional psychological and emotional health problems (e.g., negative emotions associated with conflicting cultures) because of their colonized status.

Regardless of the conflicting cultures that the young women said they experience, the young women in this study demonstrated what the known sponsor and I believe to be a high level of resiliency. As stated in the results section, one young women explained how she told herself over and over that she was not going to let other people make her hate herself. Therefore, although she was faced with the conflicting cultures, she explained how she was confident with her body and therefore able to feel good about her body. As stated by Werner (1984), resilient youth know that they can overcome the odds and it seemed as though this young woman had overcome the odds.

The young women in this study possess many attributes that have been associated with resiliency. In addition to confidence, the young women in this study had set very clear future goals and were optimistic about the future. Benard (1991) described how optimism about the future is a characteristic associated with resilient youth. All of the young women in this study had academic goals that went beyond a high school education. Although they had all experienced some degree of adversity throughout their lives, this did not limit their goals that they had set for their future. 
In addition to the belief that there are conflicting cultures, the young women in this study spoke about the importance of belonging and fitting comfortably within a society or a community, which has been defined by numerous researchers (e.g., Hewitt, 2000; Rotheram \& Phinney, 1987; Striegel-Moore \& Cachelin, 1999) in a variety of ways (i.e., social identity, ethnic identity, group identity). Hewitt (2000) defined this need to belong as social identity because the identity is anchored in a sense of belonging and being able to identify with a group of people with which one feels comfortable. Similarly, Rotheram and Phinney (1987) refered to this need to belong to a particular ethnic group as ethnic identity. They described how an individual's thoughts, feelings, perceptions, and behaviours are influenced by a sense of belonging to an ethnic group. As noted in the results section, one participant explained how she felt better when her family moved to an area with "more native people...my own people". She said how she was more "comfortable and more happy", possibly because she felt like she belonged when she was surrounded by people from her own ethnic group. Furthermore, StriegelMoore and Cachelin (1999) described how group identity could help to promote positive self-views of appearance. Findings from this study are consistent with those of StriegelMoore and Cachelin because one young woman explained how she felt better about herself (i.e., positive self-view) when she no longer had to worry about fitting in with white people.

Whether we call the need to belong social identity, ethnic identity, or group identity, an individual may experience positive emotions when they feel that they can identify with their community of choice (Hewitt, 2000; Rotheram \& Phinney, 1987; Striegel-Moore \& Cachelin, 1999). However, as noted in the results section, some young Aboriginal women may lack this feeling of belonging because they do not feel 
that they can ever belong to the white society within which they live. This lack of membership, or not feeling like they belong to the community or ethnic group within which they live, may in turn lead to a feeling of incompleteness (Hewitt, 2000). This feeling of incompleteness of not being able to belong to a certain group may be a result of the conflicting cultures and not feeling they fit the standards of any group.

Although the young women in this study noted the importance of belonging to a group (e.g., social identity), they also noted the importance of having their own personal identity. The value of possessing both identities has been supported by Hewitt (2000) who said that it is important to have a balance between personal identity and social identity. Although the perceptions of one's group will influence how one views the self, perceptions of one's self are also very important (Smith, Walker, Fields, Brookins, \& Seay, 1999). The young women recognized that they wanted to belong to a group or a community; however, they also knew that they were different and that everyone has their own unique characteristics.

Once again this speaks to this notion of resiliency. Regardless of the different beliefs that they often face, the young women were able to remain autonomous, a trait that Benard (1993) associates with resiliency. The importance of autonomy was apparent throughout the study. Although the young women noted the importance of belonging, the young women often spoke about the importance of personal identity. One participant described how important it is to have individuality, and she expressed her belief that beauty comes in a variety of shapes, colours, and sizes through her artwork and poem.

The young women in this study spent a lot of time speaking to the importance of personal identity, and identity has been recognized in the "Indigenist" Stress-Coping 
model as a central component in the coping process because it can work as a cultural buffer to social experiences (Walters \& Simoni, 2002). The young women were asked about the strengths that they felt they possessed as young Aboriginal women. As stated in the results section, one participant described how Aboriginal people are more "in tune" with their spirits. Although it was never explicitly said by any of the participants, it is possible that this spirituality is unique to those who identify as Aboriginal. Furthermore, this spirituality may act as a cultural buffer (i.e., spiritual coping; Walters \& Simoni, 2002) in how they cope with and experience specific emotions. For example, the spirituality of the young women may help them to cope with certain stressors (i.e., discrimination), which in turn may help to mitigate the damage on psychological and emotional health (i.e., anxiety, depression). In addition, although the young women said that they sometimes feel that they do not belong to either culture, they also noted how they sometimes feel comfort in the fact that they do identify themselves as Aboriginal. Consistent with the "Indigenist" Stress Coping model, Aboriginal identity can help the young women to cope with certain life stressors and therefore Aboriginal identity may foster a sense of reassurance for these young women.

The recognition of the importance of personal identity is also consistent with feminist theory in that the concept of the "universalized" woman is fading (Olesen, 2000). The words of the young women showed that they respect the differences among all young women. Also, as previously stated, some of the participants in this study explained how "everyone comes in different shapes and sizes", which is consistent with Sherwood and Neumark-Sztainer (2001) who found that many young adolescent and preadolescent girls recognize and are comfortable with the fact that young women come in a variety of shapes. Over $96 \%$ of the young women in Sherwood and Neumark- 
Sztainer's study said that pretty girls come in a variety of sizes and shapes and attractiveness cannot be equated with just one size or one particular type of person.

While recognizing that beauty comes in many forms, the young women of this study also recognized that there is variety among Aboriginal women and that not all Aboriginal women look or experience things the same. While discussing the belief that there is not just one Aboriginal identity, it is important to discuss the work of Abrams and Stormer (2002). Consistent with the findings from this study, they found that ethnicity is not a fixed risk factor for body-related emotions. More specifically, they found that those young women with ethnically heterogeneous peer groups would experience different levels of awareness and internalization of social standards. This could explain why there were sometimes discrepancies among the beliefs held by the young women in this study. Just because most of the young women identified themselves as Aboriginal, it did not necessarily mean they had the same beliefs or experienced things the same. Although Abrams and Stormer recognized the impact that ethnicity has on the body-related emotions, they recognized that ethnicity must be viewed as a dynamic factor that is dependant on numerous factors (i.e., peer groups). Therefore, even though individuals may be of the same ethnicity, it does not necessarily mean that they will experience things the same. Once again, the findings of this study are supportive of feminist theory. As suggested by Collins (1986), feminist theory recognizes that race, class, and gender are interrelated and contributing factors to the overall experiences of young women. When analyzing the data, it became apparent that the unique experiences of these young women were comprised of not only their ethnic and/or racial identification (i.e., Aboriginal, white) but also by their class and gender. Although all of the participants were women, and three of them identified themselves as 
Aboriginal, the young women all described unique experiences throughout the study. As stated by the known sponsor, it is possible that the young women have unique experiences because of the various socio-economic status or social class that they fit within.

Despite their uniqueness, the young women made numerous references to the variety of ways in which the body-related emotions can directly affect health, which has been supported in the literature (i.e., Cash, 2002; French et al., 1994; Friedstad \& Klepp, 1997; Harter, 1999; Leary, 1995; Page et al., 1993; Page \& Fox, 1997, Rubin, Numeroff, \& Russo, 2002). With regards to mental health, the young women in this study explained how negative emotions could cause people to be "jealous" or "vindictive", and thereby be detrimental to overall mental health. The experiences of the participants fit well within Cognitive-Motivational-Relational Theory because, as described by Lazarus (1991), we often experience jealousy when there is goal incongruity or when we cannot achieve something. Therefore, as stated by the participants, some young women are jealous because they cannot look a certain way.

Cash (2002) noted similar findings in that those individuals who experience negative emotions surrounding their body will typically report lower self-esteem. More specifically, he explained how one who dislikes their body would have a difficult time liking oneself. When describing physical health, one participant described how she was tempted to stay on drugs because they helped her to stay slim. This finding has been supported by numerous researchers (e.g., French et al., 1994; Friedstad \& Klepp, 1997; Harter, 1999; Leary, 1995; Page et al., 1993; Page \& Fox, 1997) who have found that concerns surrounding the body have been associated with numerous health compromising behaviours. 
This notion that the body affects everything is consistent with Rubin et al. (2002) who found that body consciousness could be damaging to an individual's mental health and physical health. Some of the young Aboriginal women in this study described how when they were younger they would experience negative emotions because they did not feel like their physical traits would ever allow them to look like white people. This finding is supported by Cognitive-Motivational-Relational Theory and self-presentation theory in that young women may be motivated by specific goals (e.g., to look like a white person) or may be motivated to present a desired impression but negative emotions are experienced when perceived goals or impressions can not be met (Lazarus, 1991; Leary \& Kowalski, 1995).

Aside from the direct affect on health, the young women also stated how the body and how one looks will sometimes influence who they are friends with and who is represented in the media, which could then have a negative affect on mental health. The young women in this study said that although they recognize that beauty comes in a variety of shapes and sizes, they also recognize the social ramifications of associating with people who do not equate with society's beauty standards. One participant explained how she would not want to be seen with a "massively overweight person" because other people may not want to associate with her.

In addition to the social implications, and similar to the results of Aidman (1999), some of the young women in this study recognized the lack of media representation of Aboriginal women. They felt that young Aboriginal women did not fit the media created ideal image of blonde hair and blue eyes, and therefore, felt that they were not represented in the media. As stated in the results section, because it is not common to see Aboriginal women in fashion magazines and other forms of media, this 
can make young women feel "tiny" and "small". Although the young women said that these images could make them feel "tiny" and "small", their overall reaction to the images was not overly negative. It is possible that the resiliency and confidence of these young women have helped them to reject this notion of the media portrayed "ideal beauty". Nevertheless, it seems that the media and those individuals who are depicted in the media, play a direct role in the overall mental health of young Aboriginal women. Sherwood and Neumark-Sztainer (2001) have supported this notion that the media affects the mental health of young women. Although these researchers do not feel that the media causes unhealthy emotions surrounding the body, they do feel that media exposure reinforces these negative emotions. 


\section{CHAPTER 4}

\subsection{SUMMARY AND CONCLUSIONS}

Research has provided inadequate insight into the body-related emotional experiences of young Aboriginal women. Although quantitative literature has shown that young Aboriginal women can experience negative emotions surrounding the body, very little descriptive qualitative research has been conducted. Furthermore, although some have recognized the strengths of using a feminist perspective when examining issues surrounding the body, young Aboriginal women have seldom been provided the opportunity to share their body-related emotional experiences. Therefore, this research project attempted to fill the gaps in the literature by utilizing case study methodologies to provide insight into the body-related emotional experiences of young Aboriginal women. Cognitive-Motivational-Relational Theory was used as the conceptual framework, while feminist theory was used as the guiding perspective.

This study involved four young women. Through the use of multiple methods (i.e., focus group, one-on-one interviews, art project) the young women were able to share the emotions that they were experiencing with regards to their body. The information that was gathered by working from within this feminist perspective has allowed for an in-depth view into the experiences of these young women. The five themes that emerged from the data included: conflicting cultures, need to belong, personal identity, journey to acceptance, and the body affects everything. The stories of 
the young women in this study differed from some of the most recent quantitative literature that states young Aboriginal women are often dissatisfied with their body. All of the young women in this study noted a general level of satisfaction or happiness with their bodies. Furthermore, the results of this study demonstrated the complexity, and context specificity, of the emotions that were experienced.

\subsubsection{Limitations}

The strengths of this study have been demonstrated in the previous sections. However, as with all research, there are some accompanying limitations to the study. This section will begin with a brief overview of the limitations associated with feminist perspective and Cognitive-Motivational-Relational Theory. This will be followed by a discussion of the methodological limitations.

It seemed only natural to utilize feminist theory as the guiding perspective for this study because Shildrick and Price (1999) have stated, "feminism has long seen its own project as intimately connected to the body" (p. 1). Although many of the results of this study are supportive of working within a feminist perspective there are some difficulties that were faced when working within this perspective. First, there are many definitions and interpretations of feminist perspective and, as a result, there is no distinct path for feminist researchers to follow. Feminist perspective allows for research that is varied and emergent and thus many debates arise over the efficacy of this research. Some researchers (e.g., Harding, 1987) believe that there is no such thing as distinct feminist methods. Similarly, some feminist researchers (e.g., Madriz, 2000) are comfortable with the fact that all feminist researchers do not take one unified approach. Once it was decided that this research would be approached using a feminist perspective, 
it was important to decide upon my own personal feminist beliefs and work from within this perspective. Although I had chosen the perspective that I felt comfortable working within, the difficulties that I faced have been summed up best by Olesen (2000) who stated that feminist qualitative research is "highly diversified, enormously dynamic, and thoroughly challenging to its practitioners, its followers, and its critics" (p.215). Feminist perspective was chosen because of its dynamic and emergent nature; however, its dynamic nature also presents challenges and limitations to future researchers who have no unified method to follow.

In addition to the controversies associated with specific definitions of feminist research, Gillies and Alldred (2002) have identified a number of feminist researchers who disagree with the idea of white women trying to speak for and represent women of colour. They described how feminist researchers should only try to represent those individuals from within their immediate communities. Therefore, some feminist researchers would disagree with my work with young Aboriginal women because I identify myself as white and I was trying to represent the experiences of the particiants. Although I feel that I was able to gain trust, it is possible that I may have been better able to understand these young women if I were Aboriginal. Nevertheless, if I am to be consistent with my own feminist beliefs, I believe that we all experience things differently regardless of racial identification. Researcher bias is inherent in qualitative representations of participants and one of the strengths of qualitative research is that each researcher brings his/her own perspective (Creswell, 1998). Furthermore, although all women are different, the participants and I shared many similarities seeing as we are all women who live in an urban center. The stories of these young women deserved to be 
told; and although I brought a non-Aboriginal perspective to this study, I believe that it is still an important perspective.

Although feminist theory was utilized as the guiding perspective, CognitiveMotivational-Relational Theory was utilized as the conceptual framework. This study supports Cognitive-Motivational-Relational Theory in that emotion is a complex context driven process in which appraisal and coping are the two key components. However, although the young women offered some suggestions as to how they cope with their emotions, they admitted that this was not something that they usually, if ever, thought about. Throughout the interviews it was apparent that the young women were coping with multiple emotions; nevertheless, the young women had a difficult time trying to articulate some of the specific strategies they use. Therefore, although CognitiveMotivational-Relational Theory was useful in that it provided direction for the types of questions that should be asked, the coping strategies of the young women may not be something that they are consciously aware of. This made it difficult to use CognitiveMotivational-Relational Theory as the conceptual framework because the process of emotion was not as clear to the participants as the theory seems to suggest.

Lazarus (1991) described how there are discrete emotions that individuals will experience. Although the young women recognized that they were experiencing various emotions, they often had a difficult time trying to identify the specific emotions that they experienced because of the complex nature of these emotions. Lazarus (1991) provided an exhaustive list of the emotions that a person can experience; however, the young women sometimes found it difficult to narrow their experiences down to one specific emotion. The young women were usually able to articulate their emotions; however, the participants used broad terms such as satisfaction to describe how they felt, as opposed 
to a discrete emotion (i.e., happy) that Lazarus described. It is possible that the emotions that were described by Lazarus were not part of the "everyday" language that the young women used. Lazarus' theory was not developed based on the language of young women; therefore, it is possible that the emotions described by Lazarus are not part of the young women's familiar discourse. Regardless, in order to be consistent with feminist perspective, it was important to represent the young women by using their own terms instead of forcing the language used by Lazarus. Although the young women did not use the terms described by Lazarus, their emotion process was consistent with Cognitive-Motivational-Relational Theory. Therefore, although somewhat limited, this theory helped to better understand the experiences of these young women.

Aside from the limitations associated with the guiding perspective and conceptual framework, there were also some methodological limitations that were faced due to the qualitative nature of the study. Although I chose to investigate this issue using qualitative research, I recognized the limitations associated with this type of inquiry. Feminist researchers, especially those using qualitative inquiry, often avoid the search for a "unified voice" of women (Wilson \& Rosenburg, 2002). This may be problematic to those researchers who are striving to implement change because they need to know whether or not the findings can be generalized to larger populations. Although the goal of qualitative research is often not to generalize but to transfer findings to other situations with similar characteristics (Lincoln \& Guba, 1985), Yin (1989) writes how some qualitative research (i.e., case studies) may be generalized. This point may be important for future researchers who wish to utilize findings from this study to initiate change. 
There were also other limitations that were associated with the qualitative nature of this study. First, quite a large time commitment was required from the participants. This time commitment was discussed prior to the start of the research so that the participants were aware of the time frame of the study. Nevertheless, two of the participants were unable to complete the art project due to other commitments that required their time. One young woman was preparing for the birth of her child and another young woman had other family commitments.

Another limitation to this study was the issue of confidentiality. All of the young women in this study were informed about the importance of confidentiality; however, the participants could only be assured a certain level of confidentiality. The participants took part in a focus group and, thus, were exposed to the experiences of the other young women. Because the participants were already familiar with each other prior to the study, it is with hope that they respected the confidential nature of our discussions.

In addition to the above-mentioned limitations, some researchers may feel that the researcher bias that is inherent in qualitative research may act as a limitation. Although it is not only qualitative research that is influenced by researcher bias, some feel that this bias plays a larger role in qualitative research. Much of the results section is based on my interpretation of the emerging themes and therefore my own experiences have helped to form the results. However, by writing my own personal story prior to the methods section I hoped to provide the readers of this thesis with an accurate representation of the strengths and biases that I was able to bring to this study. Furthermore, by member-checking the themes with the participants I was able to feel comfortable representing the participants. 


\subsubsection{Recommendations for Future Research}

Throughout the remainder of this section, a brief description of six recommendations for future research will be provided. Based on my research findings, I feel that there is still a need for more descriptive qualitative research. Although five themes emerged from the multiple phases that were conducted in this current study, the body-related emotional experiences of young Aboriginal women have rarely been researched and it is important to gain a more in-depth understanding of their experiences. Young Aboriginal women must be provided the opportunities to share their experiences because it is their stories that will help us to better understand their bodyrelated emotional experiences.

In addition to needing more qualitative research, there is a need for more research that utilizes multiple methods of inquiry especially when researching sensitive topics such as the body and its associated emotions. The body-related emotional experiences of the young women from this study were private issues that were sometimes difficult to explain. One young woman explained how she is "really in touch" with her emotions but she said she is often hesitant to express them. Interestingly, when asked why, she said that she was more self-conscious about her emotions than she was of her physical body. Although only one young woman voiced this concern of being self-conscious about her emotions, it is possible that the other three young women felt the same. Nevertheless, through the multiple methods of inquiry (i.e., focus group, oneon-one interviews, art project) the young women did try to articulate their emotions. The different phases built upon one another and provided the young women with the opportunity to share their experiences in different ways. By the end of the study the young women felt as though they were able to express their emotions; this speaks to the 
importance of developing rapport and trying to understand participant meaning through multiple methods.

Once we have a more in-depth understanding of the body-related emotional experiences of young Aboriginal women, it is important to try to understand how common these body-related emotional experiences are among a larger population of young Aboriginal women. Because of the descriptive nature of qualitative research, there is often a trade off in the depth of the research for the breadth of research (Patton, 2002). Qualitative case study methods have been useful in this current study for beginning to uncover the complexity and uniqueness of the various body-related emotions experienced by young Aboriginal women. However, a small sample size was utilized in this current study and future researchers should build upon this study to determine how generalizable these findings are to a larger population of young Aboriginal women.

Once we know how common these issues are, future researchers need to begin to work towards change. Olesen (2000) described how feminist research often sets the stage for other research, action, and change. Creswell (1998) described how feminist research draws attention to the needs of people in attempt to identify areas in need of change and the young women in this study identified what needed to be changed. They said that there should be "rallies towards freedom of the body". Heilman (1998) has identified schools as possible sites for implementing this change. She has said that educators can play an important role in addressing the social forces that often lead to distorted identity formation and thus negative body-related emotional experiences. She further explained how schools could help young women to understand their own emerging personal identities without outside influence (i.e., media, family). Heilman 
further noted how schools could play a particularly important role for young women in that educators can help to foster power and instill confidence. Although action can be implemented many ways within the schools, she has made numerous recommendations. Some of these recommendations include the need for teaching and counseling that is informed by an understanding of the effect of media, gender, class, and ethnicity. Furthermore, schools could provide support systems that try to eliminate social, gender, and class differences.

In addition to the need for action research, there is also a need for the development of a theoretical model that explains the process of the body-related emotional experiences of young Aboriginal women. Although some models, such as the model of self-presentational processes proposed by Leary and Kowalski (1990), focus on the body-related experiences of young women, they do not account for the various cultural influences on individual experiences. For example, the self-presentation model proposes that individuals try to present particular impressions of themselves and these impressions are influenced by motivation and the images that they want to foster. However, this model does not speak to the importance of ethnic or cultural identity in the self-presentation process. The words of the participants indicate that ethnic or cultural identity is an important factor in the self-presentation process. For example, young women are often influenced and motivated to present certain images (i.e., media portrayed "ideal beauty") but they often want to identify with their own cultural or ethnic group by sharing similar physical traits. However, because ethnic or cultural identity (i.e., dark hair and dark skin) often contrasts with what the media portrays as beautiful, it is possible that these young women could experience negative emotions. 
A model that does address the importance of identity attitudes with regards to individual experience is the "Indigenist" Stress-Coping Model (Walters \& Simoni, 2002). However, although this model is effective in its ability to explain how identity is important in the coping process of life stressors in general, it does not specifically address the role of identity with regards to its impact on the body-related emotional experiences of young Aboriginal women. Therefore, future researchers should consider developing a new model that incorporates the strengths of previous models to explain the process of the body-related emotional experiences of young Aboriginal women.

Lastly, future researchers should put more emphasis on the literature that examines young women's developmental changes surrounding perceptions of the physical-self. Current research has shown that there are numerous developmentally based changes that occur throughout adolescence with regards to individuals' selfperceptions (Horn, 2004). However, as noted by Horn (2004), most of this research has been published in the psychology literature and few researchers have focused on the developmental trends with regards to adolescents' perceptions of their physical-self. It is possible that a focus on developmental research may help to provide a better understanding of the body-related emotional experiences of young Aboriginal women, especially in terms of the journey to acceptance. Few researchers have tried to understand this process of acceptance that young women experience, and future researchers may be able to provide insight into this journey if more focus is placed on developmental trends. 


\section{REFERENCES}

Abrams, L. S., \& Stormer, C. C. (2002). Sociocultural variations in the body image perceptions of urban adolescent females. Journal of Youth and Adolescence, 31, 443-450.

Aidman, A. (1999). Disney's Pocahontas: Conversations with Native American and Euro-American girls. In S. R. Mazzarella \& N. O. Pecora (Eds.), Growing up girls: Popular culture and the construction of identity (pp. 133-158). New York: P. Lang.

Allison, M. T. (1988). Breaking boundaries: Future directions in cross-cultural research. Leisure Sciences, 10, 247-259.

Alway, J. (1995). The trouble with gender: Tales of the still-missing feminist revolution in sociological theory. Sociological Theory, 13, 209-228.

Ashcroft, B., Griffiths, G., \& Tiffin, H. (2000). Post-colonial studies: The key concepts. London: Routledge.

Battiste, M., A., \& Henderson, J. H. (2000). Protecting Indigenous knowledge and heritage: A global challenge. Saskatoon, SK: Purich.

Baumgartner, T. A., \& Strong, C. H. (1998). Conducting and reading research: In health and human -performance (2 $2^{\text {nd }}$ ed.). New York, NY: McGraw-Hill Companies, Inc. 
Bayer, B. M., \& Malone, K. R. (1996). Feminism, psychology, and matters of the body. Theory and Psychology, 6, 667-692.

Benard, B. (1991). Fostering resiliency in kids: Factors in the family, school, and community. Portland, OR: Western center for drug free schools and communities.

Benard, B. (1993). Fostering resiliency in kids. Educational Leadership, 51, 44-48.

Bogdan, R. C., \& Biklen, S. K. (1998). Qualitative research for education: An introduction to theory and methods ( $3^{r d}$ ed.). Boston: Allyn and Bacon.

Cash, T. F. (2002). Women's body images. In G. M. Windgood \& R. J. DiClemente (Eds.), Handbook of women's sexual and reproductive health: Issues in women health (pp.175-194). New York: Kluwer Academic/Plenum Publishers.

Centers for Disease Control and Prevention (CDC) (2000). National Center for Health Statistics CDC growth charts: United States. Retrieved March 15, 2004, from www.cdc.gov/growthcharts.htm.

Collins, P. H. (1986). Learning from the outsider within: The sociological significance of black feminist thought. Social Problems, 33, 14-32.

Creswell, J. W. (1998). Qualitative inquiry and research design: Choosing among five traditions. Thousand Oaks, CA: Sage Publications.

Creswell, J. W. (2003). Research design: Qualitative, quantitative, and mixed methods approaches $\left(2^{\text {nd }}\right.$ ed.). Thousand Oaks, CA: Sage Publications.

Crocker, P. R. E., Kowalski, K. C., Graham, T. R., \& Kowalski, N. P. (2002). Emotion in sport. In J. M. Silva \& D. E. Stevens (Eds.), Psychological foundations of sport. Boston, MA: Allyn and Bacon. 
Cummins, J. C., Ireland, M., Resnick, M., \& Blum, R. M. (1999). Correlates of physical and emotional health among Native American adolescents. Journal of Adolescent Health, 24, 38-44.

Denzin, N. K. (2000). The practices and politics of interpretation. In N. K. Denzin \& Y. S. Lincoln (Eds.), Handbook of qualitative research ( $2^{\text {nd }}$ ed., pp. 897-922). Thousand Oaks, CA: Sage Publications.

Denzin, N. K., \& Lincoln, Y. S. (1994). Handbook of qualitative research. Thousand Oaks, CA: Sage Publications.

Denzin, N. K., \& Lincoln, Y. S. (2000). Handbook of qualitative research $\left(2^{\text {nd }}\right.$ ed.). Thousand Oaks, CA: Sage Publications.

Dittmar, H., Lloyd, B., Dugan, S., Halliwell, E., Jacobs, N., \& Cramer, H. (2000). The "body beautiful": English adolescents' images of ideal bodies. Sex Roles, 42, $887-915$.

Edwards, R. (1996). White woman researcher-black women subjects. In S. Wilkinson \& C. Kitzinger (Eds.), Representing the other: A feminism \& psychology reader (pp. 83-88). Thousand Oaks, CA: Sage Publications.

Eklund, R. C., \& Crawford, S. (1994). Active women, social physique anxiety, and exercise. Journal of Sport \& Exercise Psychology, 16, 431-448.

Esterberg, K. G. (2002). Qualitative methods in social research. Boston, MA: McGraw Hill.

Figueira-McDonough, J. (1998). Environment and interpretation: Voices of young people in poor inner-city neighborhoods. Youth \& Society, 30, 123-163. 
Fine, M. (1994). Working with the hyphens: Reinventing self and other in qualitative research. In N. K. Denzin \& Y. S. Lincoln (Eds.), Handbook of qualitative research (pp.70-82). Thousand Oaks, CA: Sage Publications.

Flegal, K., Carroll, M., Ogden, C., \& Johnson, C. (2002). Prevalence and trends in obesity among US adults, 1999-2000. Journal of the American Medical Association, 288, 1723-1727.

French, S. A., Perry, C. L., Leon, G. R., \& Fulkerson, J. A. (1994). Weight concerns, dieting behavior, and smoking initiation among adolescents: A prospective study. American Journal of Public Health, 84, 1818-1820.

Frideres, J. S., \& Gadacz, R. R. (2001). Aboriginal peoples in Canada: Contemporary confilicts $\left(6^{\text {th }}\right.$ ed.). Toronto, ON: Prentice Hall.

Friedstad, C., \& Klepp, K. I. (1997). Smoking, body image and reducing diet. A threeyear follow-up of adolescents aged 15-18 years. Nordic Medicine, 112, 334-338.

Gamble, S. (2000). Postfeminism. In S. Gamble (Ed.), The routledge critical dictionary of feminism and postfeminism (pp.43-54). New York, NY: Routledge.

Gerber, L. M. (1990). Multiple jeopardy: A socio-economic comparison of men and women among the Indian, Metis and Inuit peoples of Canada. Canadian Ethnic Studies, 22, 69-84.

Gillies, V., \& Alldred, P. (2002). The ethics of intention: Research as a political tool. In M. Mauthner, M. Birch, J. Jessop, \& T. Miller (Eds.), Ethics in Qualitative Research (pp. 32-52). London: Sage Publications.

Gittelsohn, J., Harris, S. B., Thorne-Lyman, A. L., Hanley, A. J., Barnie, A., \& Zinman, B. (1996). Body image concepts differ by age and sex in an Ojibway-Cree community in Canada. Journal of Nutrition, 126, 2990-3000. 
Gruber, E., \& Ponton, L. (1995). Overweight and obesity in Native-American adolescents: Comparing nonreservation youths with African-American and Caucasian peers. American Journal of Preventative Medicine, 11, 306-310.

Hanley, A. J., Harris, S. B., Gittelsohn, J., Wolever, T. M., Saksvig, B., \& Zinman, B. (2000). Overweight among children and adolescents in a Native Canadian community: Prevalence and associated factors. American Journal of Clinical Nutrition, 71, 693-700.

Harding, S. (1987). Introduction: Is there a feminist method? In S. Harding (Ed.), Feminism and methodology: Social science issue (pp.1-14). Bloomington, $\mathbb{I N}$ : Indiana University Press.

Hart, E. A., Leary, M. R., \& Rejeski, J. W. (1989). The measurement of social physique anxiety. Journal of Sport \& Exercise Psychology, 11, 94-104.

Harter, S. (1999). The construction of the self: A developmental perspective. NewYork: Guilford Press.

Hewitt, J. P. (2000). Self and society: A symbolic interactionist social psychology ( $8^{\text {th }}$ ed.). Boston: Allyn and Bacon.

Heilman, E. E. (1998). The struggle for self: Power and identity in adolescent girls. Youth \& Society, 30, 182-208.

Himes, J. H., \& Story, M. (1992). Validity of self-reported weight and stature of American Indian youth. Journal of Adolescent Health, 13, 118-i20.

Horn, T. S. (2004). Developmental perspectives on self-perceptions in children and adolescents. In M. R. Weiss (Ed.), Developmental sport and exercise psychology: A lifespan perspective (pp.101-143). Morgantown, WV: Fitness Information Technology, Inc. 
James, K. (2000). "You can feel them looking at you": The experiences of adolescent girls at swimming pools. Journal of Leisure Research, 32, 262-280.

Kirmayer, L. J., Brass, G. M., \& Tait, C. L. (2000). The mental health of Aboriginal peoples: Transformations of identity and community. Canadian Journal of Psychiatry, 45, 607-616.

Lazarus, R. S. (1991). Emotion and adaptation. New York: Oxford University Press. Lazarus, R. S. (2000). Cognitive-Motivational-Relational Theory of Emotion. In Y. L. Hanin (Ed.), Emotions in sport (pp. 39-63). Champaign, IL: Human Kinetics.

Leary, M. R. (1995). Self-presentation: Impression management and interpersonal behavior. Dubuque, IA: Brown and Benchmark.

Leary, M. R., \& Kowalski, R. M. (1995). Social anxiety. New York: The Guilford Press.

Lincoln, Y. S., \& Guba, E. G. (1985). Naturalistic Inquiry. Beverly Hills, CA: Sage Publications.

MacMillan, H. L., MacMillan, A. B., Offord, D. R., \& Dingle, J. L. (1996). Aboriginal Health. Canadian Medical Association, 155, 1569-1578.

Madriz, E. (2000). Focus groups in feminist research. In N. K. Denzin \& Y. S. Lincoln (Eds.), Handbook of qualitative research ( $2^{\text {nd }}$ ed., pp. 835-850). Thousand Oaks, CA: Sage Publications.

McAuley, E., \& Burman, G. (1993). The social physique anxiety scale: Construct validity in adolescent females. Medicine and Science in Sports and Exercise, 25, 1049-1053.

McIntyre, L., Wien, F., Rudderham, S., Etter, L., Moore, C., MacDonald, N., Johnson, S., \& Gottschall, A. (2001). An exploration of the stress experience of Mi'kmaq 
on-reserve female youth in Nova Scotia. Halifax: Maritime Center of Excellence for Women's Health.

McNaughton, C., \& Rock, D. (2003). Opportunities in Aboriginal research: Results of SSHRC's dialogue on research and Aboriginal peoples. Social Sciences and Humanities Research Council of Canada.

Mi'kmaq Health Research Group (Etter, L., Moore, C., McIntyre, L., Rudderham, S., \& Wien, F.). (1999). The health of the Nova Scotia Mi 'kmaq population. Halifax: Union of Nova Scotia Indians \& Confederacy of Mainland MicMacs.

Monteath, S. A., \& McCabe, M. P. (1997). The influence of societal factors on female body image. The Journal of Social Psychology, 137, 708-727.

Morawski, J. (1990). Toward the unimagined: Feminism and epistemology in psychology. In R. Hare-Mustin \& J. Marecek (Eds.), Making a difference: Psychology and the construction of gender (pp. 159-183). New Haven, CT: Yale University Press.

Neumark-Sztainer, D., Story, M., French, S. A., Hannan, P. J., Resnick, M. D., \& Blum, R. W. (1997). Psychosocial concerns and health-comprimising behaviors among overweight and nonoverweight adolescents. Obesity Research, 5, 237-249.

Neumark-Sztainer, D., Story, M., Resnick, M. D., \& Blum, R. W. (1997). Psychosocial concerns and weight control behaviours among overweight and nonoverweight Native American adolescents. Journal of the American Dietic Asssociation, 97, 598-604.

Olesen, V. L. (2000). Feminisms and qualitative research at and into the millennium. In N. K. Denzin \& Y. S. Lincoln (Eds.), Handbook of qualitative research $\left(2^{\text {nd }}\right.$ ed., pp. 215-255). Thousand Oaks, CA: Sage Publications. 
O'Neil, J. (1986). The politics of health in the fourth world: A northern Canadian example. Human Organization, 45, 119-128.

Page, R. (1991). Indicators of psychosocial distress among adolescent females who perceive themselves as fat. Child Study Journal, 21, 203-212.

Page, A., Ashford, B., Biddle, S. J. H., \& Fox, K. R. (1993). Evidence of cross-cultural validity for the Phsyical Self-Perception Profile. Personality and Individual Differences, $14,585-590$.

Page, A., \& Fox, K. R. (1997). Adolescent weight management and the physical self. In K. R. Fox (Ed.), The physical-self: From motivation to well-being (pp. 229258). Champaign, IL: Human Kinetics.

Patton, M. Q. (2002). Qualitative research \& evaluation methods ( $3^{\text {rd }}$ ed.). Thousand Oaks, CA: Sage Publications.

Pinto, K. C. (2004). Intersections of gender and age in health care: Adapting autonomy and confidentiality for the adolescent girl. Qualitative Health Research, 14, 7899.

Piran, N. (2001). Re-inhibiting the body from the inside out: Girls transform their school environment. In D. L. Tolman \& M. Brydon-Miller (Eds.), From subject to subjectivities: A handbook of interpretive and participatory methods (pp. 219238). New York: New York University Press.

Ramazanoglu, C., \& Holland, J. (2002). Feminist methodology: Challenges and choices. London: Sage Publications.

Rand, C. S., \& Resnick, J. L. (2000). The "good enough" body size as judged by people of varying age and weight. Obesity Research, 8, 309-316. 
Reinharz, S. (1992). Feminist methods in social research. New York, NY: Oxford University Press.

Rinderknecht, K., \& Smith, C. (2002). Body-image perceptions among urban Native American youth. Obesity Research, 5, 315-327.

Rosen, L. W., Shafer, C. L., Dummer, G. M., Cross, L. K., Deuman, G. W., \& Malmberg, S. R. (1988). Prevalence of pathogenic weight-control behaviors among Native American women and girls. International Journal of Eating Disorders, 7, 807-811.

Rotheram, M. J., \& Phinney, J. S. (1987). Introduction: Definitions and perspectives in the study of children's ethnic socialization. In J. S. Phinney \& M. J. Rotheram (Eds.), Children's Ethnic Socialization: Pluralism and Development. California:Sage Publications.

Royal Commission on Aboriginal Peoples (1996). Report of the Royal Commission on Aboriginal Peoples. Ottawa: The Commission.

Rubin, L. R., Fitts, M. L., \& Becker, A. E. (2003). "Whatever feels good in my soul": Body ethics and aesthetics among African American and Latina women. Culture, Medicine and Psychiatry, 27, 49-75.

Rubin, L. R., Nemeroff, C. J., \& Russo, N. F. (2002). Exploring feminist women's body consciousness. Psychology of Women Quarterly, 28, 27-37.

Sarto, G. E. (1998). How race, ethnicity, and culture influence women's health. Women's Health in Primary Care (Supplement), 1, 7-14.

Schlenker, B. R., \& Leary, M. R. (1982). Social anxiety and self-presentation: A conceptualization and model. Psychological Bulletin, 92, 641-669. 
Sherwood, N. E., \& Neumark-Sztainer, D. (2001). Internalization of the sociocultural ideal: Weight-related attitudes and dieting behaviors among young adolescent girls. American Journal of Health Promotion, 15, 228-231.

Shildrick, M., \& Price, J. (1999). Openings on the body: A critical introduction. In J. Price \& M. Shildrick (Eds.), Feminist Theory and the Body: A reader (pp.1-14). New York: Routledge.

Smith, L. T. (1999). Decolonizing methodologies: Research and indigenous peoples New York: Zed Books.

Smith, E. P., Walker, K., Fields, L., Brookins, C. C., \& Seay, R. C. (1999). Ethnic identity and its relationship to self-esteem, perceived efficacy and prosocial attitudes in early adolescence. Journal of Adolescence, 22, 867-880.

Stake, R. E. (1995). The art of case study research. Thousand Oaks, CA: Sage Publications.

Stein, J. S. (1997). Empowerment and women's health: Theory, methods, and practice. London: Zed Books.

Story, M., French, S.A., Resnick, M.D., \& Blum, R.W. (1995). Ethnic/racial and socioeconomic differences in dieting behaviors and body image perceptions in adolescents. International Journal of Eating Disorders, 18, 173-179.

Story, M., Hauck, F. R., Broussard, B. A., White, L. L., Resnick, M. D., \& Blum, R. W. (1994). Weight perceptions and weight control practices in American Indian and Alaska Native Adolescents. Archives of Pediatrics and Adolescent Medicine, $148,567-571$.

Striegel-Moore, R. H., \& Cachelin, F. M. (1999). Body image concerns and disordered eating in adolescent girls: Risk and protective factors. In N. G. Johnson, M. C. 
Roberts \& J. Worell (Eds.), Beyond appearance: A new look at adolescent girls (pp. 85-108). Washington, DC: American Psychological Association.

Sweeney, M.L. (2001). The self-image of adolescent females: A group exploration. In K.A. Fall \& J.E. Levitou (Eds.), Modern applications to group work (pp. 69-96). Huntington, NY: Nova Science Publishers Inc.

Thompson, J. L., Allen, P., Cunningham-Sabo, L., Yazzie, D. A., Curtis, M., \& Davis, S. M. (2002). Environmental, policy, and cultural factors related to physical activity in sedentary American Indian women. Women \& Health, 36, 59-74.

Vasquez, M. J. T., \& Fuentes, C. (1999). American-born Asian, African, Latina, and American Indian adolescent girls: Challenges and Strengths. In N. G. Johnson, M. C. Roberts, \& J. Worell (Eds.), Beyond appearance: A new look at adolescent girls (pp. 151-173). Washington, DC: American Psychological Association.

Walters, K. L., \& Simoni, J. M. (2002). Reconceptualizing Native women's health: An "Indigenist" Stress-Coping Model. American Journal of Public Health, 92, 520524.

Way, N. (2001). Using feminist research methods to explore boy's relationships. In D. L. Tolman \& M. Brydon-Miller (Eds.), From subjects to subjectivities: $A$ handbook of interpretive and participatory methods (pp. 111-129). New York: New York University Press.

Werner, E. (1984). Resilient children. Young Children, 40, 68-72.

Wilson, K., \& Rosenberg, M. W. (2002). Exploring the determinants of health for First Nations peoples in Canada: Can existing frameworks accommodate traditional activities? Social Science \& Medicine, 55, 2017-2031. 
Wright, B. A. (1991). Labeling: The need for greater person-environment individuation. In C. R. Snyder \& D. R. Forsyth (Eds.), The handbook of social and clinical psychology: The health perspective (pp. 474-475). New York: Pergamon Press.

Yin, R. K. (1989). Case study research: Design and methods. Thousand Oaks, CA: Sage Publications.

Yin, R. K. (1994). Case study research: Design and methods (2 ${ }^{\text {nd }}$ ed.). Thousand Oaks, CA: Sage Publications. 
$\vec{F}$

昜 


\section{APPENDIX A}

Ethical Approval 


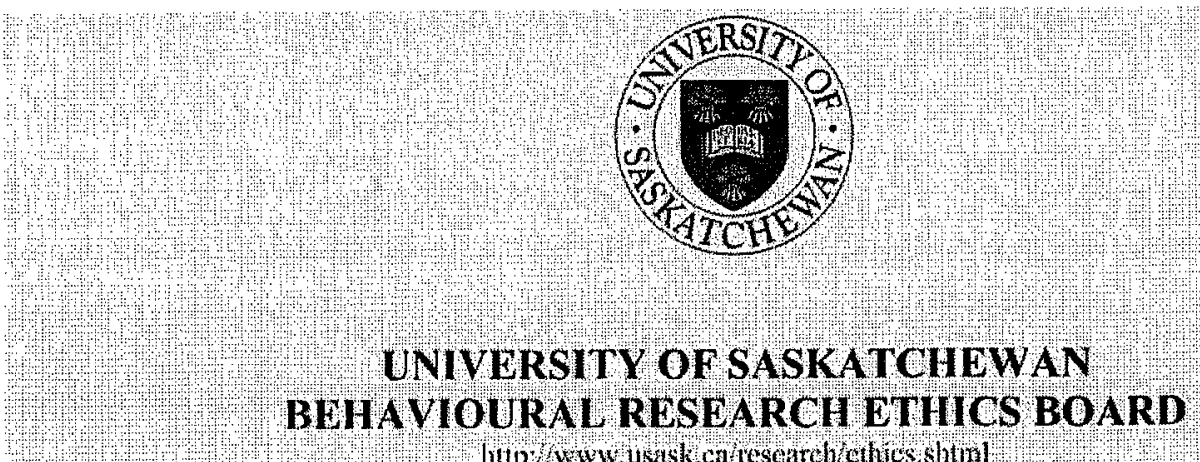

hith: wow wosk catescareh chics shemi

NAME: Kent Kowalski (Tara-Leigh Flerning)

BSC\#: 03-1346

College of Kinesiology

DATE: Deember 12,2003

The University of Saskatchewan Beliavioural Research Ethics Board has reviewed the Application for tothics Approval for your study "A Qualitative Study of the Body-Related Emotional Experiences of Young Aborignal Woment $(03-1346)$.

1. Your study has been APPROVED.

2. Any significant changes to your proposed method, or your consent and recruitment procedures should be reported to the Chair for Committee consideration in advanee of its implementation.

3. The term of this approval is for 1 year.

4. This approval is valid for one year $A$ status report form nust be submitted annually to the Chatr of the Committee in order to extend approval. This certificate will automatically be invalidated if a status teport form is not received within one month of the anniversary date. Plase refer to the website for further instructions http/www usask ca/research behavrse.shtm

I wisl yon a suecessful and informative study.

Dr David Hay, Acting Chair Unversity of Siskatchewan

Behavioural Research Ethies Board

DHuck

Office of Research Services, University of Saskatchewan

Room 1607, 110 Gymaslum Place, Box 5000 RPO Universily, Saskatoon SK S7N 4JB CANADA

Telephone (306) 966-8576 Facsimile (306) $966-8597$

http//www usask ca/research 
APPENDIX B

Consent Form 


\section{UNIVERSITY OF SASKATCHEWAN}

\section{Informed Consent Form}

You are invited to participate in a study entitled: $A$ qualitative study of the body-related emotional experiences of young Aboriginal women. Please read this form carefully and feel free to ask questions you might have.

Researcher: Tara-Leigh Fleming and Dr. Kent Kowalski, College of Kinesiology, University of Saskatchewan, 966-1079

Purpose and Procedure: The purpose of the study is to provide insight into the bodyrelated emotional experiences of young Aboriginal women.

Your participation will be required in four phases. Each phase will be conducted at a time and location of your convenience, and will be led by a female researcher.

The first phase that you will be involved in will be a rapport-building phase in which we will get to know one another. We will meet, along with the other participants that you know, on a casual basis (2-3 times; approximately 45 minutes each) so that we can talk about "everyday" things and get to know more about each other.

The second phase that you will be involved in will be another rapport building session in which you will meet in a group setting, along with the other participants that you know. Within this small group, you will initially be asked to react to images seen in our culture (i.e., popular magazines). You will then take part in an art project in which you will create your own images of the body by painting, drawing, writing, and/or cutting out pictures from magazines/newspapers. You will then be asked about your choices and how you feel that your art represents your feelings about your body.

The third phase will be a one-on-one semi-structured interview in which you will be asked more specific questions focusing on some of the issues discussed in the focus groups and how you cope with your feelings about your body.

The fourth, and final phase that you will be involved in, will be another one-onone semi-structured interview used to clarify and expand emerging themes and to understand your experiences in a different way. The third interview will begin with you presenting me with some form of visual material (i.e., pictures; cultural artifacts), or with some method of expression (i.e., performance art), that represents your bodyrelated emotional experiences. You will not be judged on your method of expression, but instead you can use this time to express your feelings in a way that you feel comfortable. Your method of expression will be used to generate discussion. This final phase will also be a chance for you to provide me with any other information that you may feel is relevant to this study. You will also be asked what you feel could have been done differently in this study to try and understand individual experiences with the body. With your permission, the art projects will become the property of the researcher, although you will receive a copy. Under no circumstances will the art projects be used for financial gain.

Beginning with phase two, the phases will be audio taped, and field notes will be taken. The audio tape recordings will be transcribed (written out) word for word. As a participant, you will also be asked to provide feedback on the accuracy of interview transcripts and may be asked to review the themes that emerge from the data analysis. 
Potential Risks: You will not be subjected to any physical or psychological risk. You have the right to refuse to answer any question, at which time the discussion will be redirected. In the event that you would like to discuss your feelings about the questions raised in the interview further, a referral can be made to the appropriate counseling services. Should we feel that your continuation in the project is placing you under undo stress (e.g., establishing mutual times for interview sessions), we can choose to discontinue your involvement in the study, at which time any data that has been collected will be deleted (erased) from the study and destroyed.

Potential Benefits: Participation may help develop a better understanding of the bodyrelated emotional experiences of young Aboriginal women. The results of the study may help further knowledge in this area. As well, the study might lead to ideas for intervention programs aimed at helping individuals deal with feelings about their bodies. These benefits are not guaranteed through participation in the study.

Confidentiality: The data from this study will be written as a Master's thesis. The data may also be published and presented at conferences; however, your identity will be kept confidential. Although we will report direct quotations from the interviews, you will be asked to choose a pseudonym (made up name) and all identifying information (name, address) will be removed from our report.

Only the research team will review the original audiotapes and transcripts. Names or other identifying particulars will not be discussed or made public outside of the research team. Audio tapes will be identified by code number and stored in a secure locked office. The audiotapes, transcripts, and photographs will be stored separately from the master sheet identifying names, pseudonyms, and code numbers.

There are, however, limits on the level of confidentiality that we as researchers can ensure. Because you will take part in a small focus group, you will be identifiable to other people in the group on the basis of what you have said. The researcher will undertake to safeguard the confidentiality of the discussion, but cannot guarantee that other members of the group will do so. Please respect the confidentiality of the other members of the group by not disclosing the contents of this discussion outside the group, and be aware that others may not respect your confidentiality. After your interview, and prior to the data being included in the final report, you will be given the opportunity to review the transcript of your interview, and to add, alter, or delete information from the transcripts as you see fit. Also, it is important that you are aware that there are certain types of information that the researcher may be obliged to report to relevant authorities (e.g., child abuse, intent to do violence).

Storage of Data: All research material will be securely stored in the office of Dr. Kent Kowalski, at the University of Saskatchewan, for a minimum of five years post publication of the findings.

Right to Withdraw: You may withdraw from the study for any reason, at any time, without penalty of any sort, including current or future participation in this or any other program. Should you decide to withdraw from the project, your information will be deleted (erased) from the study and destroyed. 
Questions: If you have any questions concerning the study, please feel free to ask at any point; you are also free to contact the researcher at the number provided above if you have questions at a later time. Any questions regarding your rights as a participant may be addressed to the University of Saskatchewan Behavioural Sciences Research Ethics Board through the Office of Research Services (966-4053). This study was approved on

You may contact the researcher to find out the results of the study or request to be involved in the review the themes that emerge from the data analysis. A copy of the published manuscript can also be requested.

Consent to Participate: I have read and understood the description provided above. I have been provided with an opportunity to ask questions and my questions have been answered satisfactorily. I consent to participate in the study describe above, understanding that I may withdraw this consent at any time. A copy of this consent form has been given to me for my records.

only:

I give permission for the art projects to be used under the following conditions as raw data, not to be viewed outside of the research team , only those projects that do not reveal my identity may be used for educational purposes (professional and research presentations) and research publications, all projects can be used for educational purposes (professional and research presentations) and research publications.

Participant's Name Participant's Signature

Researcher's Name $\quad$ Researcher's Signature

Parental/Guardian Consent: My signature on this sheet indicates that I have received information regarding the nature of the study, its purpose, and procedures, and I will allow my daughter, qualitative study of the body-related emotional experiences of young Aboriginal women.

Signature of parent or guardian Date 


\section{APPENDIX C}

Interview Guide: Reaction to Media Images 


\section{Interview Guide: Reaction to Media Images}

1. Tell me what you think of the images in these magazines

2. What kinds of images are depicted in these magazines/media?

3. How attainable are these images to young women?

3a. How important is it to be like these women?

3b. How are women in society portrayed?

4. What kind of effect do these images have on women of different ethnicities (i.e., Aboriginal)?

5. What are some of the strengths of being Aboriginal that are not captured in these images?

5a. What does it mean to be Aboriginal?

6. What other forms of media influence people?

7. What are some of the main body-related issues that youth are dealing with? 


\section{APPENDIX D}

Interview Guide: Focus Group 


\section{Interview Guide: Focus Group}

1. Did you enjoy creating the artwork?

2. Describe what the artwork means to you?

3. How does the artwork represent how you feel about the body?

4. Is there a piece of this artwork that best describes your body?

5. Is there a specific colour corresponding to the artwork that you feel represents how you feel about your body? 


\section{APPENDIX E}

Interview Guide: One-on-one Interview 


\section{Interview Guide: One-on-one Interview}

Ask specific questions pertaining to artwork. Ask participants to expand on certain aspects of their artwork.

1. Sometimes people feel that they are being watched, and maybe judged, by their peers, can you recall a time when this may have happened to you?

2. How would you describe your body?

2a. What words would you use to describe your body?

2b. How do you feel about your body?

2c. How did you get to feel this way about your body? (prompt: family, peers, media...ask for examples)

3. When are you most comfortable with your body or with people looking at your body?

4. When are you least comfortable with your body or with people looking at your body?

5. How do you know people are looking at your body?

5a. How do you feel when you know people are looking at your body? -Can you describe what it is that makes you feel uncomfortable?

-What is at stake for you? (i.e., why do you care?)

$5 \mathrm{~b}$. Who is looking at your body?

6. What do you do when you know your body is being watched or judged by others?

6a. What other strategies do you use?

6b. How do you decide to use some strategies over others?

-Do you have some examples? 
6c. Are there any reasons that you chose certain strategies as opposed to other strategies?

6d. What type of strategies do your friends use?

6e. What type of strategies do other people use? (Do you approve of these strategies?)

7. Can you describe the things that are important for you to show through your physical appearance?

8. How close are you to achieving that?

9. Are there certain things you wear that make you feel a certain way? (if they give an example of something that makes them feel good, ask for an example of something that does not make them feel good and vice versa)

10. Throughout this interview we have touched on a number of issues relating to your experience with your body. Before we finish the interview, is there anything more that you would like to add to what you have already shared about your experience with your body? 


\section{APPENDIX F}

Participant Artwork 


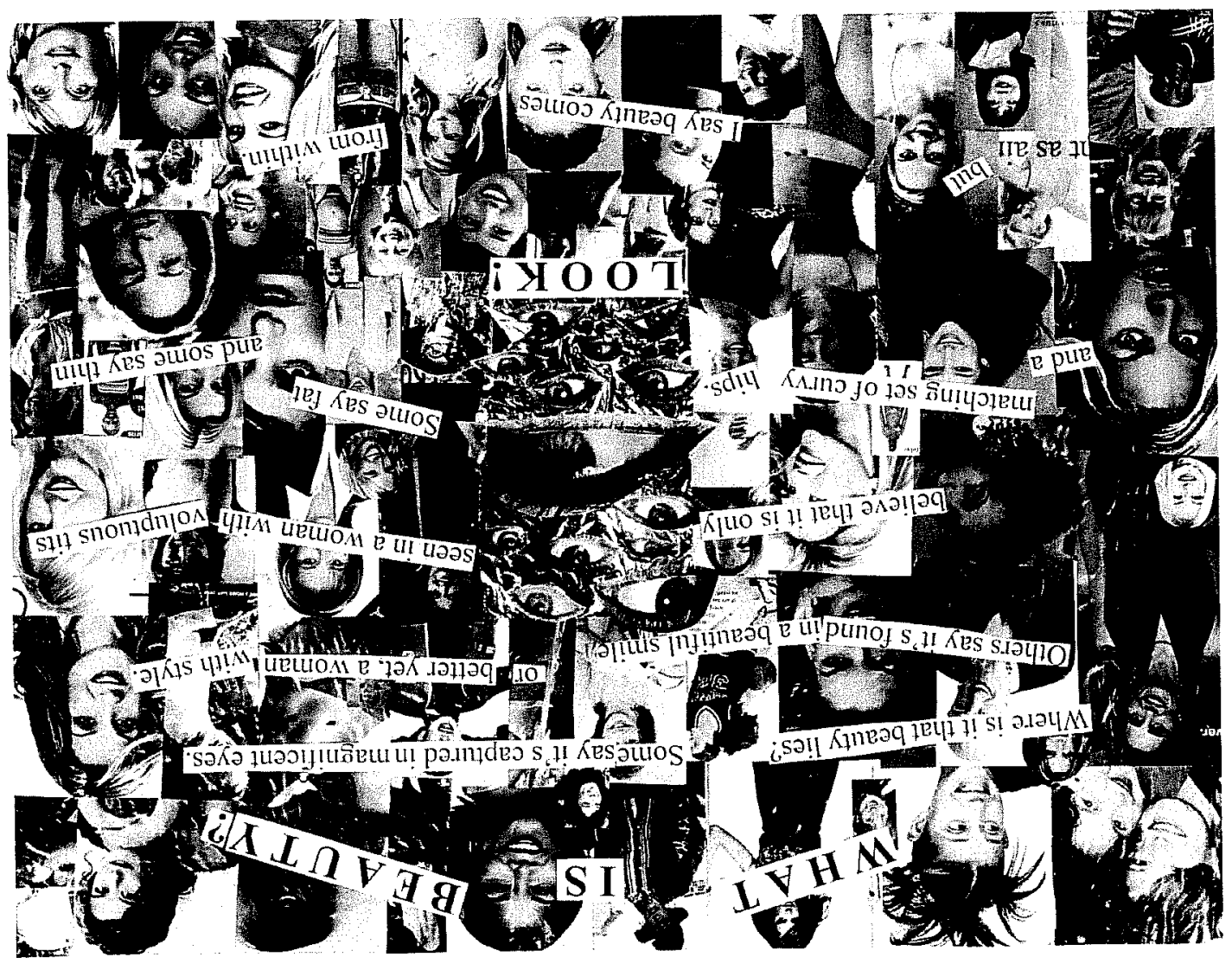




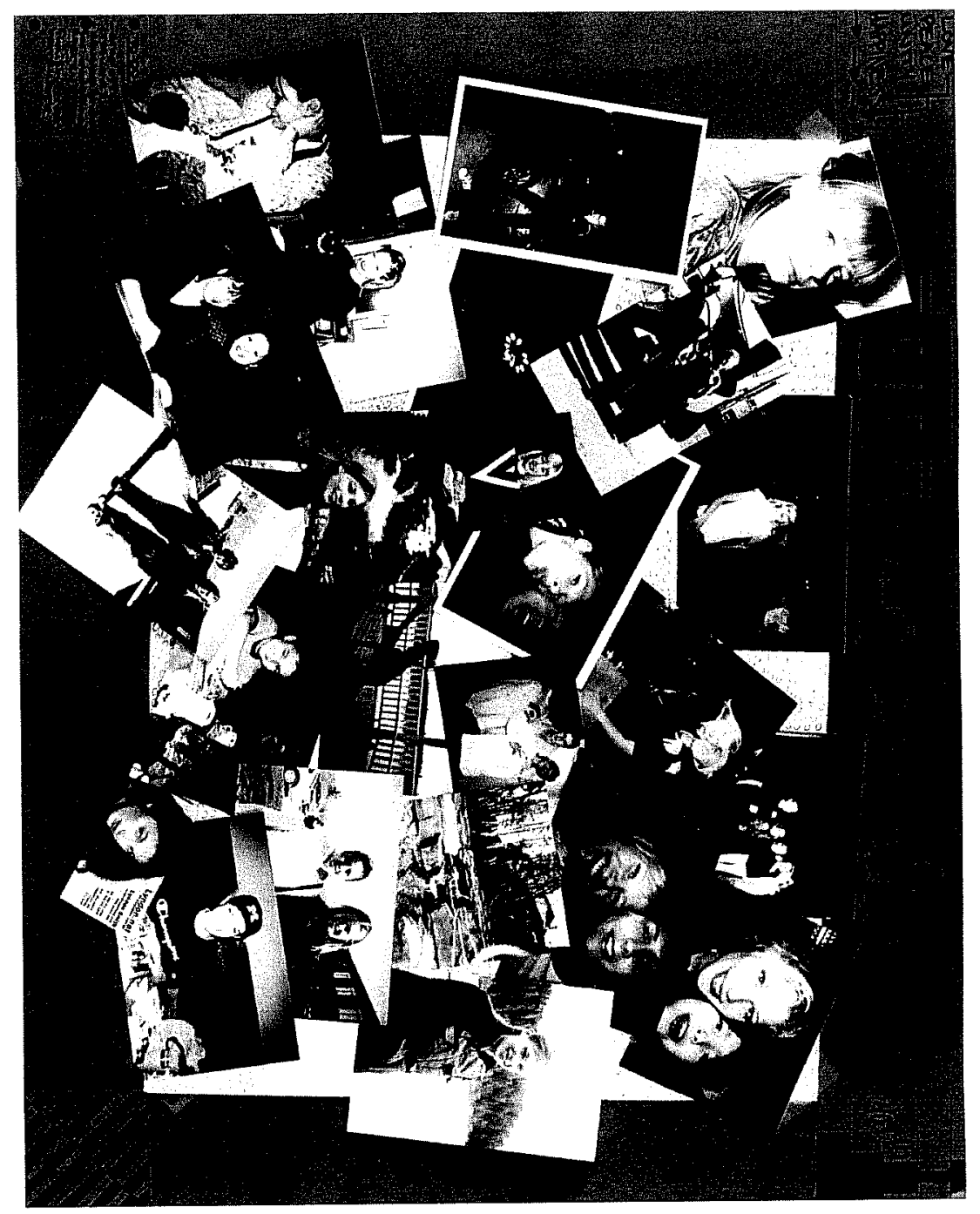




\section{APPENDIX G}

Interview Guide: Artwork 


\section{Interview Guide: Artwork}

1. Tell me about your artwork

2. How does this artwork represent the specific theme that you chose to represent?

3. How did you choose this type of art (i.e., collage)?

4. How does this artwork represent how you feel about your body? 


\section{APPENDIX H}

Critique Checklist for a Case Study Report

(Stake, 1995, p.131) 


\section{Critique Checklist for a Case Study Report}

1. Is the report easy to read?

2. Does it fit together, each sentence contributing to the whole?

3. Does the report have a conceptual structure (i.e., themes or issues)?

4. Are its issues developed in a serious and scholarly way?

5. Is the case adequately defined?

6. Is there a sense of story to the presentation?

7. Is the reader provided some vicarious experience?

8. Have quotations been used effectively?

9. Are headings, figures, artifacts, appendixes, and indexes used effectively?

10. Was it edited well, then again with a last-minute polish?

11. Has the writer made sound assertions, neither over-nor-under-interpreting?

12. Has adequate attention been paid to various contexts?

13. Were sufficient raw data presented?

14. Were data sources well chosen and in sufficient number?

15. Do observations and interpretations appear to have been triangulated?

16. Is the role and point of view of the researcher nicely apparent?

17. Is the nature of the intended audience apparent?

18. Is empathy shown for all sides?

19. Are personal intentions examined?

20. Does it appear that individuals were put at risk? 